\title{
Experimental and numerical study of the separation angle for flow around a circular cylinder at low Reynolds number
}

\author{
By MING-HSUN WU ${ }^{1}$, CHIH-YUNG WEN ${ }^{2}$, \\ RUEY-HOR YEN ${ }^{3}$, MING-CHENG WENG \\ AND AN-BANG WANG ${ }^{1} \dagger$ \\ ${ }^{1}$ Institute of Applied Mechanics, National Taiwan University, No. 1, Roosevelt Rd., \\ Sec. 4, Taipei, Taiwan 106, Republic of China \\ ${ }^{2}$ Department of Mechanical Engineering, Da-Yeh University, Chang-Hwa, Taiwan 51505, \\ Republic of China \\ ${ }^{3}$ Department of Mechanical Engineering, National Taiwan University, No. 1, Roosevelt Rd., \\ Sec. 4, Taipei, Taiwan 106, Republic of China
}

(Received 30 April 2002 and in revised form 30 April 2004)

The separation point of the flow around a circular cylinder has been numerically and experimentally investigated in the regime of Reynolds number less than 280 . The present results reveal that the long-existing discrepancy in the data concerning the time-averaged separation angles reported in the literature results mainly from the oscillating characteristics of the flow separation on the cylinder surface and the experimental methodologies rather than the commonly mentioned blockage-ratio effect. In the present experiment, the time-averaged separation angles are obtained by averaging the instantaneous images from a soap-film flow visualization instead of from the commonly used streakline images from finite time exposures. Excellent agreement has been achieved between the present experimental results and numerical simulations by the spectral element method. Particle-streak visualization in a towing tank has also been conducted to compare with that of the two-dimensional soap-film experiments. It reveals that the separation angle is insensitive to the three-dimensional effect. Variations of the time-averaged separation angles with Reynolds number can be represented by a four-term $\theta_{s}-R e^{-1 / 2}$ relationship in the range of $7 \leqslant R e \leqslant 200$. Moreover, if the data in the very low Reynolds number region are excluded, a simple linear $\theta_{s}-R e^{-1 / 2}$ relationship can be derived for $10 \leqslant R e \leqslant 200$. Since the dimensionless boundary layer thickness and the Strouhal-Reynolds number relationship for the circular cylinder are also known to be proportional to $R e^{-1 / 2}$, this linear relationship offers direct evidence that the flow characteristics of the boundary layer extend downstream along the cylinder surface to the separation point in this $R e$-range. The blockage effect on the separation angle has also been quantitatively analysed.

\section{Introduction}

The vortex shedding phenomena associated with flow separation from the boundary layer of a circular cylinder have attracted the interest of scientists and engineers for a great many years. Among the large number of investigations, some studies have

$\dagger$ Author to whom correspondence should be addressed: abwang@spring.iam.ntu.edu.tw 


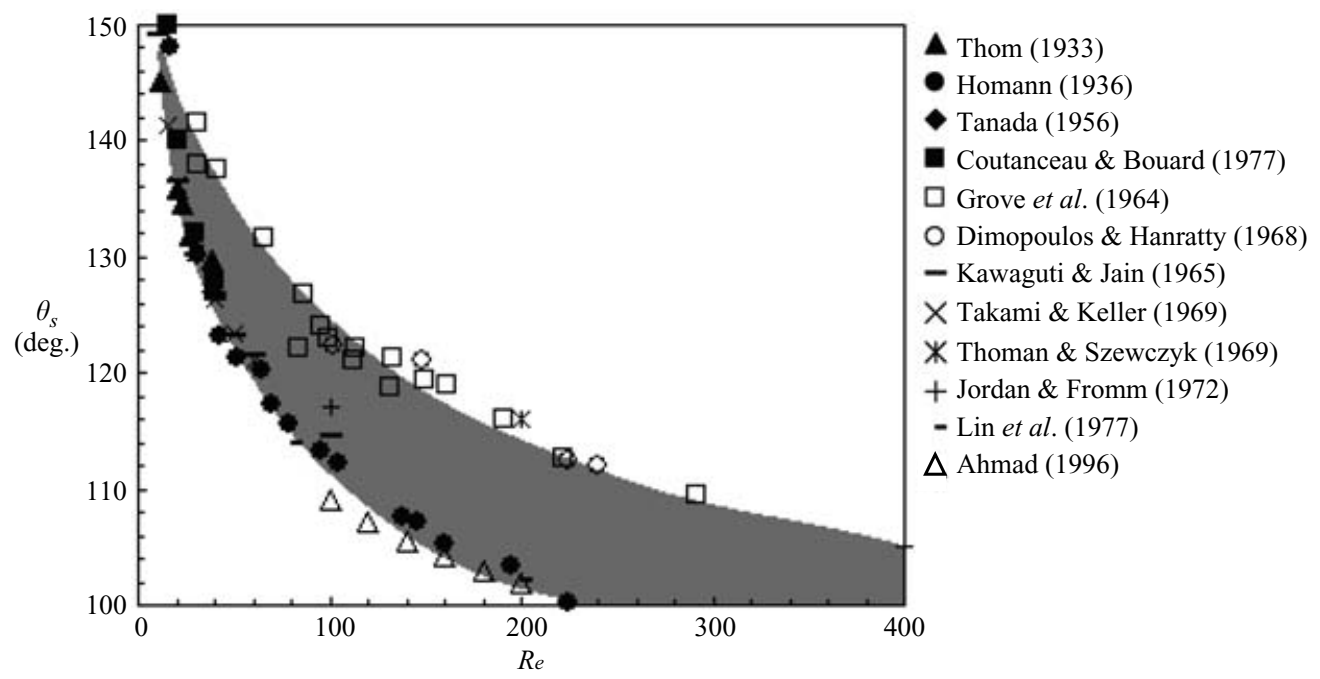

FIGURE 1. Relationships of separation angle and Reynolds number from different authors, among which a clear deviation band, shown as a grey region, exists.

focused on the separation angles in the laminar flow regime, since the flow structure around the separation point has been recognized as the origin of vortical instabilities in the wake. However, it is surprising that, as shown in figure 1, the existing data in the literature concerning the separation angle of the flow around a circular cylinder do not agree with each other. There is an approximately $10^{\circ}$ deviation band (shown as the grey area) for the observed separation angles among various researchers. The deviation becomes larger when the Reynolds number increases. The discrepancy seems especially significant in a regime in which unsteady wakes behind the circular cylinder occurs. It is worth emphasizing here that almost all the existing experimental and numerical data are located at the edge of the grey band. From the results in figure 1, it is really difficult to say which data are 'the most correct'. Thus, there is confusion in declaring that the numerical result is consistent with that of the experiment. This motivated the present researchers to initiate this study in order to determine the origin of the vortex shedding phenomena. Whether there is a bifurcation in the separation angle for flow around the cylinder in this regime or whether there are parameters such as unclear systematic errors in different experimental and numerical methods, the commonly mentioned blockage ratio of the test model and the three-dimensionality of the flow need to be addressed.

For an experimental study of the cylinder wake, streakline visualization, especially by continuous release of tracers (dye, smoke, bubbles, electrochemical material, etc.) from the cylinder wall, is the most commonly used method in the literature, since vorticity is generated from the solid boundary. For example, Thom (1933) investigated this problem by an ink-injection technique, and Grove et al. (1964) used both the bubble-tracer technique and variation in the refractive index due to the change in the density caused by slightly heating the cylinder. Dimopoulos \& Hanratty (1968) employed an electrochemical technique to probe the separation point of the boundary layer on the cylinder. Due to the restriction of the visualization methods and the strong reflection of the illuminating light from the cylinder wall, the separation point is commonly obtained by an extrapolation of the observed streakline from the boundary of the recirculation bubbles behind the cylinder. 
All researchers observed the averaged separation angles directly from finite-timeexposed photographs (i.e. time-averaged images), although the actual separation criterion should follow the basic definition that the instantaneous wall-shear stress is zero. However, as indicated by many authors, e.g. Merzkirch (1974) and Coutanceau \& Defaye (1991), vorticity diffuses considerably more rapidly than tracers, and the observed tracer filament contains information that is integrated all along the path of the tracers from the point of introduction. This might lead to misinterpretation of the corresponding recorded patterns, especially for the unsteady wake flow when $R e>R e_{C}$, where $R e$ is the Reynolds number and $R e_{C}$ is the critical Reynolds number for the onset of vortex shedding. The Reynolds number is defined as $R e \equiv U D / v$, where $U$ is the free-stream velocity, $D$ is the diameter of the circular cylinder, and $v$ is the kinematic viscosity of the working fluid. As a result, it is uncertain whether the images of streakline patterns result from the flow distortion or the tracer memory.

Another visualization method is to observe the instantaneous streamline patterns. The paths of individual particles that are randomly dispersed within the fluid initially are recorded for a very short time. Compared to streakline-pattern visualization, the streamline method has the advantage of giving direct access to the velocity field and information concerning the flow topology. Homann (1936) and Taneda (1956) used this method by adding aluminium powder to the fluid to form streamline patterns of near wakes and show the structural evolution of the flow with the Reynolds number. Difficulties also arise in streamline-pattern interpretations in the case of unsteady flows and free-surface flows that may be affected by interfacial effects and the non-identical pressure condition.

In the foregoing review, all the flow visualizations were implemented in threedimensional flow facilities. None of these experiments adopted end-manipulating methods. In recent experiments, simple parallel end-disks (or end-plates) have been widely used at both ends of the cylinder to provide controllable and symmetric end conditions and isolate the central test region from unwanted lateral disturbances. The aspect ratio, defined as the cylinder length between the end-disks to the cylinder diameter, has been found to be significantly influential in the shedding frequency and the critical Reynolds number for the onset of vortex shedding in the flow around a circular cylinder. Moreover, in a laminar flow regime, the vortices shed from a circular cylinder become oblique rather than parallel to the cylinder axis under the influence of the so-called end effects caused by the end-disks (Williamson 1996). Different end-manipulating methods to isolate the end effects from the test region of a cylinder have been developed (Eisenlohr \& Eckelmann 1989; Williamson 1989; Hammache \& Gharib 1991; Miller \& Williamson 1994; Wang, Trávníek \& Chia 2000). It is still unclear whether the oblique vortex shedding changes the separation angle.

Nearly two decades ago, Couder (1981) first introduced using flowing-soap films to conduct classical hydrodynamic experiments on two-dimensional flows. The thickness of these films commonly ranges from 0.1 to $10 \mu \mathrm{m}$, so that the ratio of the characteristic length of the flow structures to the film thickness routinely exceeds $10^{4}$. Thus soap films constitute the closest physical approximation to the concept of a truly twodimensional fluid. Therefore, unlike three-dimensional facilities, effects such as aspect ratios, oblique vortex shedding and three-dimensional instabilities that may influence the separation process are minimized. Recent experiments performed in soap-film devices have focused on demonstrating certain characteristics of turbulent flow that resemble those anticipated for a true two-dimensional system (e.g. Couder 1981, 1984; Couder, Chomaz \& Rabaud 1989; Gharib \& Derango 1989; Kellay, Wu \& Goldburg 
1995; Wu et al. 1995; Rutgers et al. 1996; Beizaie \& Gharib 1997; Rivera, Vorobieff \& Ecke 1998; Wen \& Lin 2001).

A complete demonstration to validate whether soap films obey the classical twodimensional Navier-Stokes equations was also presented by Chomaz (2001). A theoretical analysis of the three-dimensional soap-film dynamics is performed, using the asymptotic lubrication theory, which assumes only that the thickness of the film is small compared to the characteristic length scale of the in-plane flow. The leadingorder approximation for the dynamics of a flat soap film gives both the physics of the equilibrium in the free film and the order of magnitude of the neglected effects. The evolution equations governing the leading-order film thickness, two-dimensional velocities (locally averaged across the film thickness), average surfactant concentration in the interstitial liquid, and the surface concentration are then given. This model takes into account a large number of physical effects: film elasticity, film stiffness (curvature effect), viscosity, diffusion, arbitrary large variations of thickness, adsorption and desorption of the soap (solubility of the soap) and non-uniform initial soap concentration. A sufficient condition for the film velocity distribution to comply with the NavierStokes equations is found to be that the typical flow velocity, $U$, be small compared to that Marangoni elastic wave velocity, $U_{e}$, i.e. small Mach number, $M_{e}\left(=U / U_{e}\right)$. In that case the thickness variations are slaved to the velocity field in a very specific way that is consistent with recent experimental observations in the near wake.

One fascinating feature of flowing soap films is that, when observed in white light, they show coloured interference patterns. This feature results from a small variation in the thickness of the soap films, which can correspond to a passive scalar in the velocity field, thus providing an instantaneous and global visualization of the flow structure, such as the vorticity (e.g. Wu et al. 1995; Rivera et al. 1998; Chomaz 2001; Yang, Wen \& Lin 2001). No additional material is added to the fluid like the tracers, which, as previously mentioned, might cause problems in the interpretation of the image. This feature is expected to help determine the flow separation points. To our knowledge, there has been no direct measurement of the separation angles at consecutive instants to show the oscillating characteristics of the flow and to derive time-averaged separation angles in the low-Reynolds-number regime by using twodimensional soap-film tunnels.

For the numerical study of the cylinder wake, there are two main approaches. The first uses either a steady approach by neglecting the transient terms in the equations or a time approach, by adding an artificial false transient term, to speed up the calculations. This approach was widely adopted in the past, especially before the emerging development of computers in the 1970s and 1980s, for example, Thom (1928, 1933), Kawaguti (1953), Allen \& Southwell (1955), Keller (1958), Keller \& Takami (1966), Apelt (1958), Takami \& Keller (1969) and Nieuwstadt \& Keller (1973). Secondly, by using time-dependent calculations, Kawaguti \& Jain (1965) have shown that a steady-state solution is the limit of the non-steady-state solutions for Reynolds numbers below 50. Jain \& Sankara Rao (1969) removed the symmetry constraints in the work of Kawaguti \& Jain (1965) and obtained a periodic Kármán vortex street for $R e=60,100$ and 200 .

Thoman \& Szewczyk (1969) made extensive simulations of flow over a circular cylinder for Reynolds numbers ranging from 1 to $3 \times 10^{5}$, and Jordan \& Fromm (1972) computed the same flow for $R e=100,400$ and 1000 by using a grid of $121 \times 10^{3}$ points. They observed that the stagnation streamline and separation points oscillated in phase with the shedding eddies. Lin, Pepper \& Lee (1977) solved the flow by using finitedifference techniques for $R e=40,80$, and 200. The separation angle appears to be 
affected by the mathematical approximation of the nonlinear terms that is used to achieve numerical stability. Recently, simulations by the spectral element method have successfully predicted the different characteristics of the cylinder flows in comparison with experiments, even up to the turbulent wake transition (e.g. Henderson 1995, 1997; Blackburn \& Henderson 1999). This method has the advantage of low numerical dissipation and dispersion errors. However, in none of the systematic numerical studies using this method was an attempt made to determine the separation angles for laminar flows around a circular cylinder.

Thus, the plan of this paper is as follows. To clarify whether the discrepancies in the separation angles in the literature, as shown in figure 1, result from the experimental uncertainties or the inherent unsteady nature of the vortex-shedding phenomena, both computational and experimental approaches are adopted in the present study. In $\S 2$ and $\S 3$, brief descriptions of the numerical and experimental methods used in this study are given, respectively. On the computational side, highly accurate spectral element simulations were implemented for Reynolds numbers ranging from 5 to 200. These calculations provide an accurate determination of the time-averaged separation angles and detailed variations in the instantaneous separation angles with time for different $R e$. On the experimental side, both the soap-film method and particle-streak visualization in a towing tank were used. In $\S 4.1$, the discussion is focused on the time-varying characteristics of the separation angle. The variation in the separation angle with the Reynolds number is discussed in $\S 4.2$. The truly two-dimensional experimental results from soap-film tunnels yield a direct comparison with our twodimensional numerical calculations. The empirical relationship of the separation angle and the Reynolds number is established in $\S 4.3$, which shows that the data obtained from experiments are in excellent agreement with those from numerical simulations. The blockage effect on the separation angle is also discussed.

\section{Numerical methods}

\subsection{Governing equations and boundary conditions}

The governing equations for an incompressible two-dimensional isothermal flow over a circular cylinder are the continuity equation and the Navier-Stokes equations:

$$
\begin{gathered}
\frac{\partial u_{i}}{\partial x_{i}}=0, \\
\rho\left[\frac{\partial u_{i}}{\partial t}+u_{j} \frac{\partial u_{i}}{\partial x_{j}}\right]=-\frac{\partial p}{\partial x_{i}}+\frac{\partial}{\partial x_{j}}\left[\mu\left(\frac{\partial u_{i}}{\partial x_{j}}\right)\right]+S_{i},
\end{gathered}
$$

where

$$
S_{i}=\frac{\partial}{\partial x_{j}}\left[\mu\left(\frac{\partial u_{j}}{\partial x_{i}}\right)\right]+F_{i},
$$

and $u_{i}$ are the Cartesian velocity components, $x_{i}$ are the Cartesian coordinates, $F_{i}$ is the gravitational force, $p$ is the pressure, and $\rho$ and $\mu$ are the fluid density and viscosity, respectively. Equations (2.1) are then written in non-dimensional form: lengths are scaled by the cylinder diameter, $D$, and velocities are scaled by $U$, the magnitude of the free-stream velocity.

As shown in figure 2(a), the boundary conditions for the velocity and pressure fields are required along the exterior of the calculation domain. Uniform flow conditions are given at the inlet (on the left-hand side of figure $2 a$ ) and both top and bottom, i.e. $U^{*}=1$ and $V^{*}=0$, where $U^{*}$ and $V^{*}$ represent the dimensionless velocity in the $x$ - and 
(a)

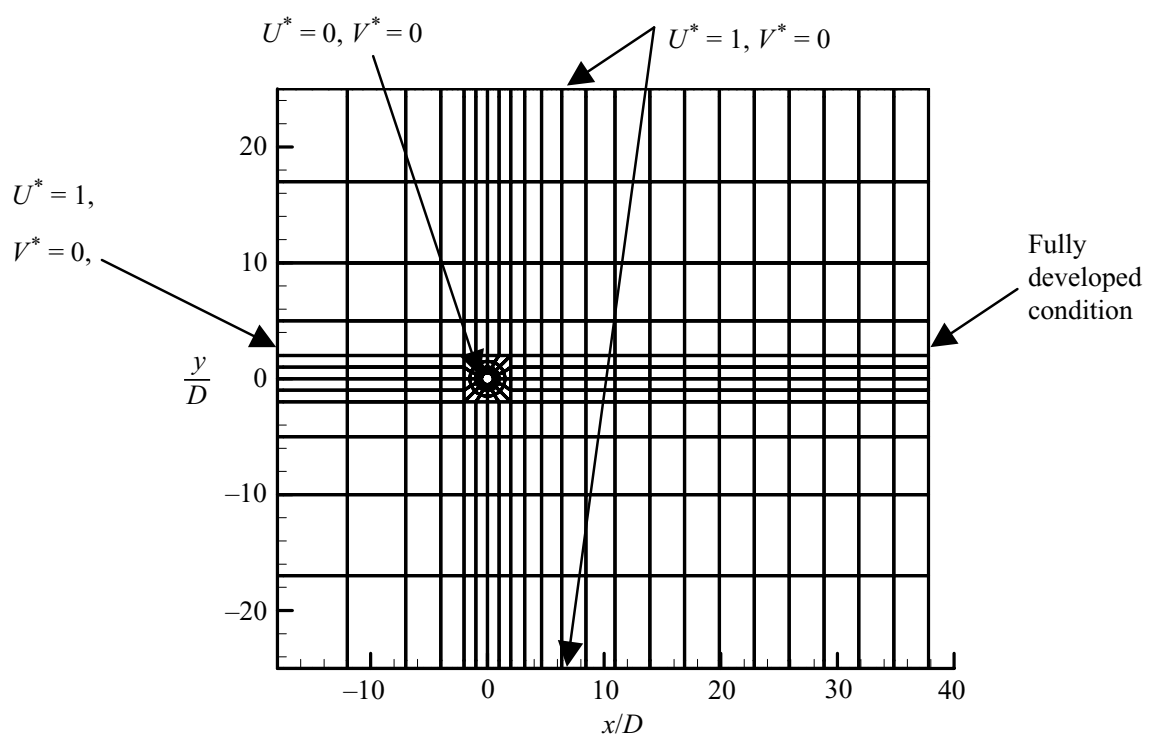

(b)

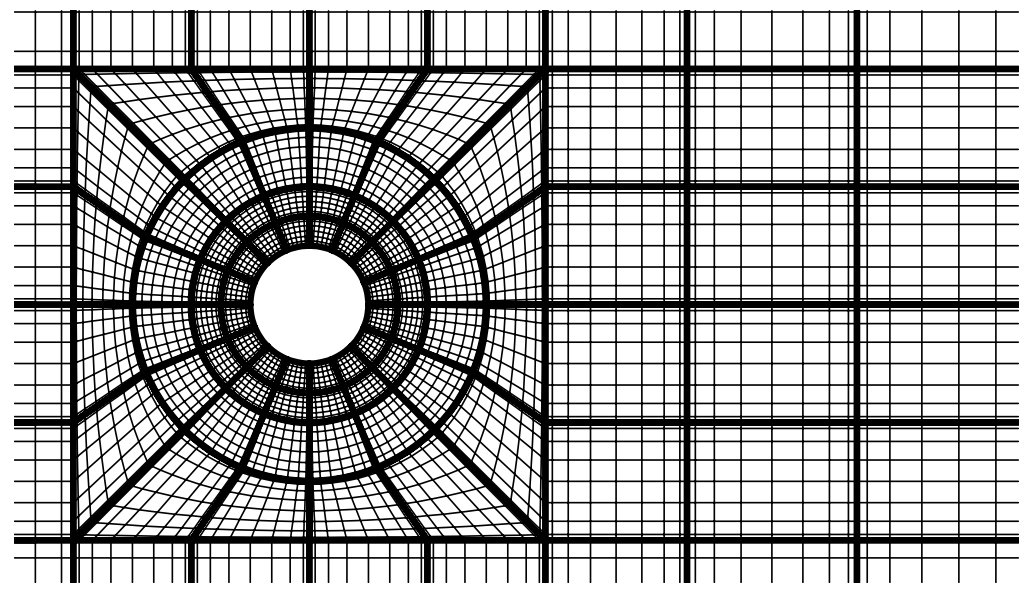

FIGURE 2. Sketch of $(a)$ computational domain, coordinate system and numerical element discretization and $(b)$ element mesh around cylinder for $9 \times 9$ nodal points (thick lines display element meshes and thin lines show grid nodal points).

$y$-directions, respectively, normalized with the free-stream velocity. A fully developed condition is used for the outflow (on the right-hand side) of the velocity. On the cylinder surface, the velocities satisfy the no-slip boundary conditions $\left(U^{*}=V^{*}=0\right)$.

\subsection{Numerical methods}

The computation was performed by the spectral-element method in space. The solutions were obtained from time-dependent simulations of the Navier-Stokes equations. The velocity and pressure coupling were treated by the second-order-mixed stiffly stable scheme in which the operator splitting proposed by Karniadakis \& Triantafyllou (1992) was incorporated. For the steady-flow calculations in the range $R e<R e_{C}$, the transient code was modified to accelerate calculations to a steady-state solution (Yen \& 
Hsu 2004). Most data in the steady recirculation regime were obtained by using this rapid steady solver.

There are three steps in solving the momentum equations. The first is to explicitly compute the forcing term, which contains the source term $S_{i}$ and the convective term $\rho u_{j} \partial u_{i} / \partial x_{j}$. As shown in (2.1), the source term in the momentum equation includes the gravitational force and the effect of variable viscosity. The second and third steps are to solve the Helmholtz equations for pressure and velocities, respectively.

The simulations were performed in the domain shown in figure 2(a), which extends 18 diameters upstream, 38 diameters downstream and 25 diameters to each side of the cylinder axis. The computational mesh, which consists of 312 elements, is similar to those used in computations by Barkley \& Henderson (1996) for the isothermal wake flow behind a circular cylinder. Figure 2(b) shows a close-up of the element mesh of $9 \times 9$ nodal points around the cylinder. Thick lines depict the element meshes and thin lines show the nodal points in the grid. To increase the resolution of the boundary layer development near the wall, the elements near the wall are much smaller than those in the outer regions. The tolerance used for the iterative conjugate gradient solvers, employed to solve the Helmholtz equations, was less than $10^{-7}$. The mass conservation in the average value of the divergence was always satisfied to $10^{-3}$.

\subsection{Derivation of separation angle and grid-independence test}

The separation point in the boundary layer is determined at the position on the cylinder wall where the shear stress is zero. The separation angle, denoted $\theta_{s}$, is measured along the streamline direction from the front stagnation point to the separation point. Since the separation angle changes with time during the vortex-shedding period, the instantaneous separation position should be derived from the instantaneous flow field. The instantaneous shear stress around the cylinder surface is calculated from the tangential velocity gradient in the radial direction of the cylinder by multiplying the fluid viscosity. All nodal points in the radial direction of the elements located closest to the cylinder wall are used for the polynomial curve fittings of the tangential velocity profiles. The velocity slopes are then derived by differentiation of the fitting curves for all grid points on the cylinder surface. If the zero shear stress point is not located precisely at the grid point, linear interpolation between these two neighbouring grid points is used to obtain the position of the zero shear stress, i.e. the instantaneous separation position. In this case, the wall-shear stresses of the neighbouring grids change sign. If there is more than one point with zero shear stress on the cylinder surface, the point closest to the front stagnation position is chosen as the instantaneous separation position. The value of the time-averaged separation angle is then derived from the arithmetic mean of typically the 18 instantaneous angles for equal time-intervals in an entire shedding period. It is worth mentioning that the value of the averaged separation angle derived from the time-averaged velocity field is slightly different from that mentioned above for $R e>R e_{C}$.

To check the numerical grid independence, seven different nodal points of $5 \times 5$, $6 \times 6,7 \times 7,8 \times 8,9 \times 9,10 \times 10$ and $11 \times 11$ grids inside each element were used for different Reynolds numbers. Figure 3 shows the numerical results of the time-averaged separation angles for different grid nodes inside an element for the cases of $R e=23$, 30,80 and 160 . The first two cases are in the steady recirculation regime, and the remaining two cases are in the laminar vortex-shedding regime. These reveal clearly that the higher the Reynolds number, the more grids per unit element are needed to obtain a grid-independent result. However, all results show that the time-averaged separation angles remain almost constant for calculations with grids of more than 


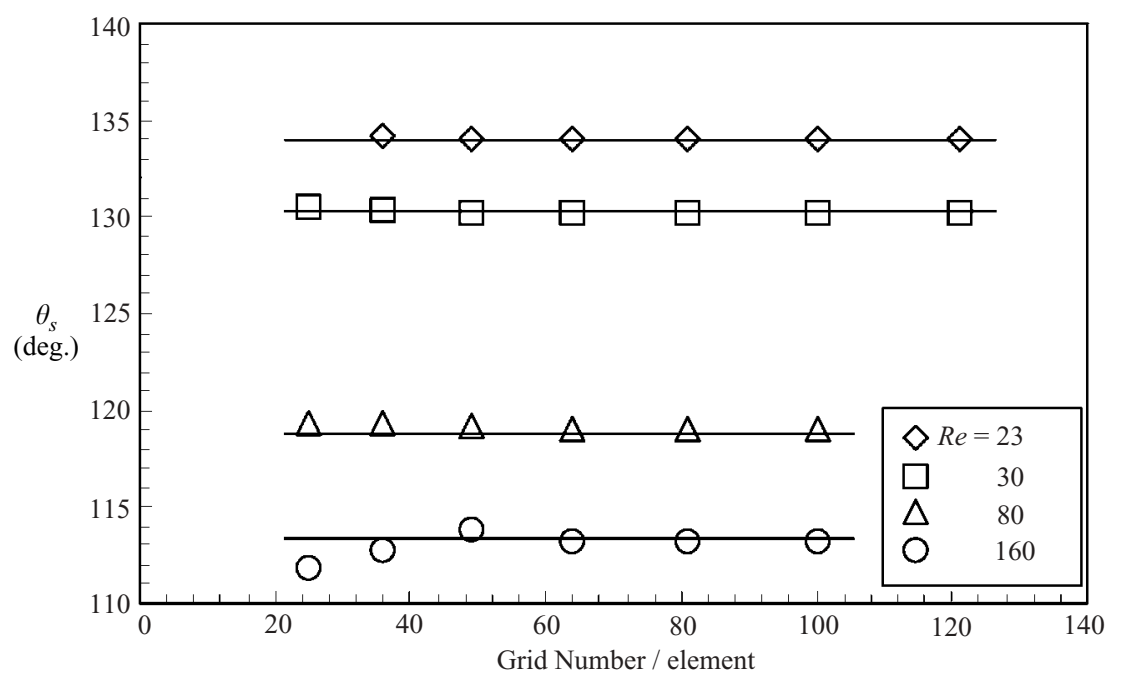

FiguRE 3. Grid-independence test of time-averaged $\theta_{s}$ values for $R e=23$, 30,80 and 160 , respectively.

$8 \times 8$. Hence, the $9 \times 9$ nodal points inside each element were chosen for all the calculations in this study.

\section{Experimental setup}

The experiments were conducted in a towing tank and two soap-film tunnels (one horizontal and one vertical for different $R e$-ranges). Since the soap-film method inherently generates a two-dimensional wake, most experimental results shown in this paper were obtained in the soap-film tunnels in order to have a direct comparison with the two-dimensional computations in $\S 2$. The results from the towing tank are used for the purpose of comparison only.

\subsection{Soap-film method}

A horizontal and a vertical soap-film tunnel were used to set up the two-dimensional flows for Reynolds numbers, Re, ranging from 20 to 275 . Note that the value 275 is much higher than the value (about 180) achievable in the traditional three-dimensional facilities, e.g. wind tunnels and water tunnels, for two-dimensional laminar flows around a circular cylinder because of the development of three-dimensional instabilities thereafter. The horizontal soap-film tunnel was originally developed by Gharib \& Derango (1989). This device consists of a frame $(5 \mathrm{~cm}$ wide and $12 \mathrm{~cm}$ long, shown at the extreme right in figure $4 a$ ) with one end positioned in a diluted soap solution reservoir, while the other end is subjected to a planar water jet as a film-pulling mechanism. The test section is the horizontal portion of the frame, consisting of two parallel copper rods supported by two legs, one at each end. The size of the frame is limited by the film's tendency to bow due to gravity in the flat section of the tunnel. The tunnel allows free-stream velocities, $U$, up to $0.9 \mathrm{~m} \mathrm{~s}^{-1}$.

The vertical soap-film tunnel depicted in figure $4(b)$ was originally developed by Kellay et al. (1995). The tunnel consists of an upper reservoir and a lower reservoir connected by two nylon fishing wires. The soap film is then bounded at its edge by these two thin lines, with the parallel vertical portion as the test section. The tunnel height is about $2.5 \mathrm{~m}$; the width (typically $5-10 \mathrm{~cm}$ ) can be changed by varying the 
(a)

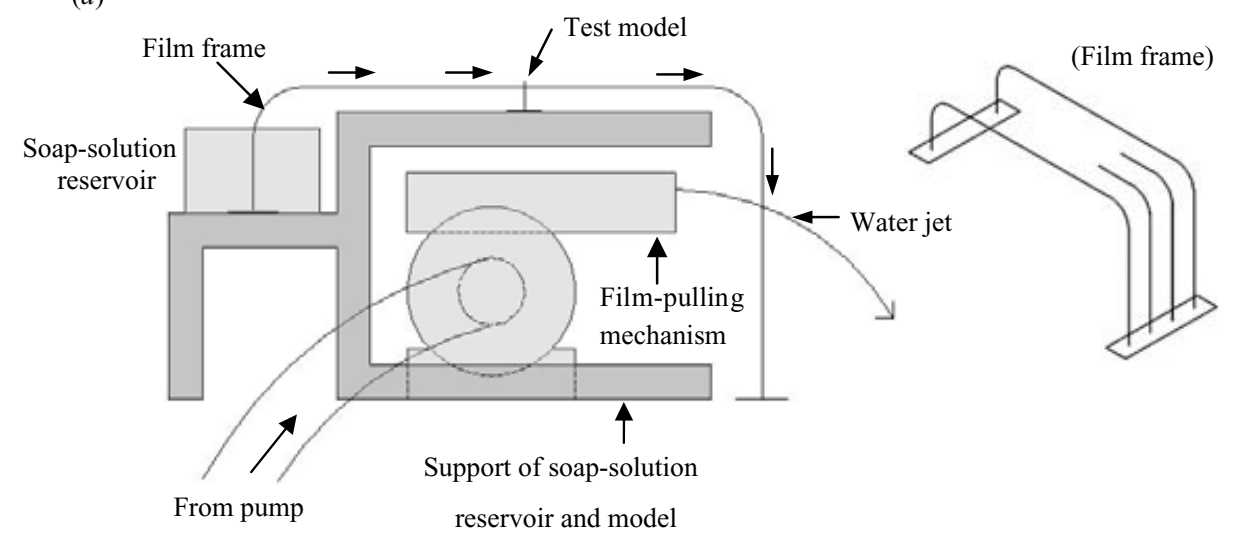

(b)

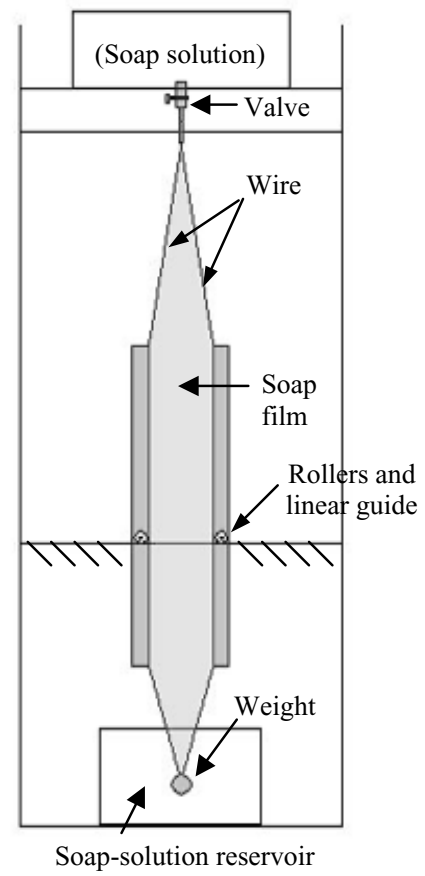

FIGURE 4. Sketch of $(a)$ horizontal and $(b)$ vertical soap-film tunnels.

separation distance between the nylon lines. The flow is driven by gravity. As the film emerges from the injection point at the top of the tunnel through a valve, it accelerates for a short distance but is then slowed by air friction across its surfaces. In the test section the film nearly approaches a terminal velocity. The free-stream velocities, ranging between 0.8 and $2.0 \mathrm{~m} \mathrm{~s}^{-1}$, can be instantly tuned by changing the injection rate of the valve. Compared to the horizontal soap-film tunnel, much larger models and higher fluid velocities are possible in the vertical tunnel and, thus, yield a higher $R e$. The vertical soap-film tunnel supplements and extends the results for the flow obtained by its horizontal counterpart. 
To ensure that soap films obey the classical two-dimensional Navier-Stokes equations, the flow Mach number, $M_{e}\left(=U / U_{e}\right)$, must be small as mentioned in $\S 1$. The Marangoni elastic wave velocity, $U_{e}$, depends on the thickness of the soap film and the composition of the soap. Taking the example of SDS (sodium dodecyl sulphate) soap molecules the waves travel at $4 \mathrm{~m} \mathrm{~s}^{-1}$ in a $10 \mu \mathrm{m}$ thick film and at $13 \mathrm{~m} \mathrm{~s}^{-1}$ in a $1 \mu \mathrm{m}$ thick film for a soap concentration of order $0.1 \%$ (see Couder et al. 1989 for details). In the current experiments, $U_{e}$, was of order $10 \mathrm{~m} \mathrm{~s}^{-1}$ and $U$ was kept below $1.5 \mathrm{~m} \mathrm{~s}^{-1}$ to ensure two-dimensionality.

Both tunnels are shielded from the room by curtains to prevent air currents from disturbing the film. The tunnels are also placed on weak-spring passive isolation systems to minimize the effects of floor vibrations. All the experiments were conducted at night when disturbances were minimal. These measures protect the film from any significant lateral motions (normal to the film surface) that impose arbitrary largescale flow on the film. Since the film is continuously fed, evaporation does not pose a problem for either tunnel.

After the film is set into motion in quiescent air, two air boundary layers grow on each side of the film. The flowing-soap film is then subject to nonlinear shear by the surrounding air in the vicinity of the cylinder. This air friction effect has been addressed in the pioneering work of Couder et al. (1989) on the hydrodynamics of soap films. The air friction will affect the film motion and consequently its twodimensionality unless the inertial force of the film element is much larger than the air friction on the film. The ratio of the inertial force to the air friction is estimated by the dimensionless parameter $C=(e \rho \sqrt{U}) /\left(2 \rho_{a} \sqrt{v_{a} D}\right)$, where $\rho$ and $e$ are the density and thickness of the film, and $\rho_{a}$ and $v_{a}$ are the density and kinematic viscosity of the air, respectively. Therefore, it is only for small diameters in thick films that the Reynolds number retains its critical role and the effect of air damping is negligible. In all our experiments, we have been careful about this issue and ensured that the air friction did not affect the film motion seriously.

Experiments were performed with mixtures of distilled water and $0.5 \%, 1.0 \%$ and $1.5 \%$ commercial liquid detergent (Ivory Dishwashing Liquid) by weight. No glycerol was added to the mixture. The film thicknesses were estimated to be 12,10 and $8 \mu \mathrm{m}$ for the flowing soap films of $0.5 \%, 1.0 \%$ and $1.5 \%$ soap concentrations, respectively, by measuring the flow rate (the discharged volume within a time period) and dividing it with the free-stream velocity and the width of the tunnel. These thicknesses represent thick soap films. Small circular cylinders with diameter $D$ ranging from 1 to $6 \mathrm{~mm}$ made of smooth stainless-steel rods were used in the experiments. The cylinder was set normal to the free stream, spanning the central part of the test section. The tunnelwall blockage ratio, $B R$, defined as the ratio of the cylinder diameter to the channel width between two tunnel walls, was kept at less than 0.08 .

A commercial one-component laser Doppler velocimetry (LDV) system (TSI IFA750 assembly) was used to measure free-stream velocities and velocity fluctuations in the cylinder wake. For both tunnels, the longitudinal turbulence intensity did not exceed $1 \%$. The free-stream velocity and turbulence intensity were uniform to within $0.007 U$ and $0.001 U$, respectively.

In order to determine the flow Reynolds number, one also needs to find the film viscosity. The dynamic viscosity of the soap film, $\mu$, has been given by Trapeznikov (1957) as $\mu=\mu_{b}+2 \mu_{s} / e$, where $\mu_{b}$ is the bulk viscosity of the interstitial fluid and $\mu_{s}$ is the surface viscosity of the superficial layers. This shows clearly that the thinner the film, the greater the contribution of the surface layers. Although Trapeznikov does not suggest any practical method to obtain the surface viscosity, from the equation 
we should expect obtain a viscosity of the soap film quite different from the bulk viscosity of water by changing the film thickness and/or the surface viscosity of the superficial layers. Due to the practical difficulty of measurement of surface viscosity and the complex properties of the commercial liquid detergents, the evaluation of the film viscosity is left to empirical methods. It is known that, for Reynolds numbers (based on the diameter of the cylinder) up to about 180, the two-dimensional vortexshedding process of a circular cylinder at a macroscopic level has a strong similarity to its three-dimensional counterpart (see Gharib \& Derango 1989 and also Wen \& Lin 2001). As a result, the vortex-shedding approach was adopted to determine the film viscosity and then the flow Reynolds number. By measuring the velocity and shedding frequency of a known-size cylinder in the film, the Strouhal number, St, can be obtained. By substituting St into Roshko's (1954) well-known equation,

$$
S t=f d / U_{\infty}=0.212-4.5 / R e,
$$

$R e$ can be found. The kinematic viscosity of the film, $v$, is determined consequently and its values are $1.32 \times 10^{-5}(e \approx 12 \mu \mathrm{m}), 2.0 \times 10^{-5}(e \approx 10 \mu \mathrm{m})$, and $3.5 \times 10^{-5}(e \approx 8 \mu \mathrm{m})$ $\mathrm{m}^{2} / \mathrm{s}$ for $0.5 \%, 1.0 \%$, and $1.5 \%$ soap-film solutions, respectively. This shows that the viscosity increases when the film thickness decreases. In the measurements of Vorobieff \& Ecke (1999), they also used the vortex-shedding approach to determine the film viscosity and found $v \approx 1.0 \times 10^{-5}$ and $3.7 \times 10^{-6} \mathrm{~m}^{2} / \mathrm{s}$ with $e \approx 3$ and $12.7 \mu \mathrm{m}$, respectively. Apparently, our soap films in this study are a little more viscous than those of Vorobieff \& Ecke (1999). Considering the differences in specific soap solutions (soap brand and concentration) and film thickness, both results show that the soapfilm viscosity can be more than 10 times that of pure water. Gharib \& Derango (1989) and Wen \& Lin (2001) performed detailed investigations of the uncertainty in the measurements of the film viscosity. The estimated uncertainty of the film viscosity was less than $\pm 5 \%$, which led to an estimated accuracy of Reynolds numbers of $\pm 6 \%$. Note that this estimated film viscosity includes the effect of all the experimental factors, such as the meniscus formation around the cylinder. Further comments about the meniscus effect are made later on.

The soap-film flows were visualized using traditional flash photography and a highspeed CCD camera. In the flash photography, the soap-film flows were illuminated by a white-light stroboscope (Strobotac 1546) and recorded by a traditional camera (Nikon FM-2). The pulse width of the light source was approximately $1.2 \mu \mathrm{s}$. A high-speed CCD camera (FASTCAM-Super $10 \mathrm{~K}$ ) was also used to freeze the images of the instantaneous flow patterns and to reveal the instantaneous oscillating flows around the test model. The resolution of each frame was set at $512 \times 240$ pixels; the frame speed was set at 500 frames per second. A close-up lens (Nikon, $200 \mathrm{~mm}$ Micro + PB 6 bellows) was added onto the CCD to magnify the flow image close to the cylinder. Illumination was provided by a halogen light source (Fostec, 8375).

\subsection{Particle-streak visualization in the towing tank}

Figure 5 shows a schematic representation of the experimental setup for the flow visualization conducted in the towing tank. A mixture of glycerol and water solution $(50 \%$ in volume) was contained in a $460 \mathrm{~mm}$ (width) $\times 455 \mathrm{~mm}$ (height) $\times 1500 \mathrm{~mm}$ (length) glass tank. The density and viscosity of the working fluid were measured before each series of experiments to accurately determine the Reynolds number. The viscosity of the glycerol-water mixture was measured by a viscometer (Brookfield, model DVII+); the density was determined by direct division of fluid weight by 


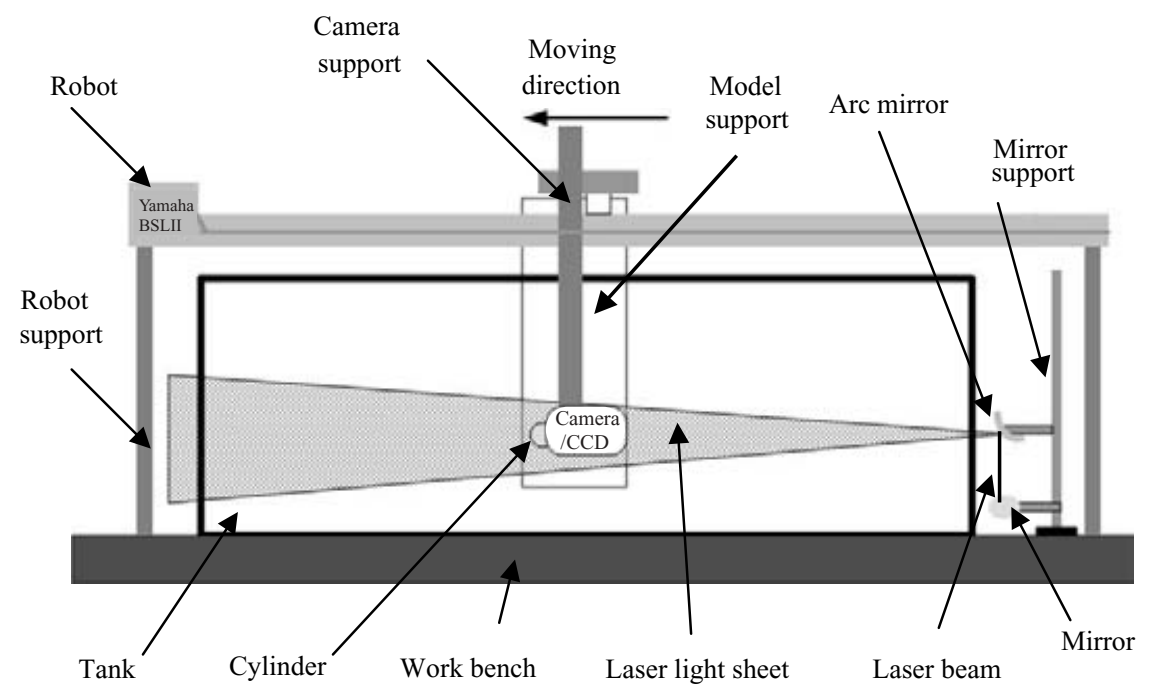

FIGURE 5. Schematic sketch of experimental setup for flow visualization in a towing tank.

volume. An electronic scales (Excell, EKW-6) was used to measure the weight; the volume was measured by a beaker.

To minimize the vibrations from the laboratory environment, the entire test section was installed on a large robust wooden desk and the particle-streak visualization experiments were conducted at night. The cylinder model was firmly fixed on a highly stable traversing unit (Yamaha, Flip-BLS II). The traversing speed ranged from $0.06 \mathrm{~cm} \mathrm{~s}^{-1}$ to $120 \mathrm{~cm} \mathrm{~s}^{-1}$. The maximum stroke of the traversing unit was $2 \mathrm{~m}$. Capillary glass tubes of different sizes were used as the model circular cylinder. The blockage ratio ranged from $1 / 18$ to $1 / 46$. The biggest capillary glass tube had an external diameter of $25 \mathrm{~mm}$. A laser sheet, of about $1 \mathrm{~mm}$ thickness, generated by a $2 \mathrm{~W}$ laser beam passing through a collimator and a cylindrical lens, was used to illuminate the flow field in the dark room. A tracer consisting of $50 \mu \mathrm{m}$ polymide particles (Dantec) was used to reveal the flow pattern. Special care was taken to minimize the image distortion in the flow observed near the cylinder. Either a digital camera (Nikon Coolpix 990) or a CCD-camera (JAI CV-M10) in conjunction with a frame-grabber (Matrox Pulsar) was used to record the image and transfer it to a computer for storage and further processing.

\section{Results and discussion}

\subsection{Time-varying characteristics of separation angle}

Figure 6 shows a series of instantaneous streamlines and vorticity lines for flow around a circular cylinder under the condition of $R e=100$ within a vortex-shedding period. The flow is from left to right. The streamlines are plotted as the solid lines and the constant-vorticity lines are shown as the dashed lines, revealing an overall view of how the streamlines and vorticity field are transformed within an entire period of vortex shedding. To acquire a better understanding of the vortex-shedding process, the flow information close to the separation point was examined. Figure 7 shows a blown-up view of the instantaneous streamlines, vorticity lines and also the velocity vectors near the upper-right quadrant of the cylinder. Nine instantaneous flow patterns of equal time intervals in a complete vortex-shedding period are displayed in this figure. 


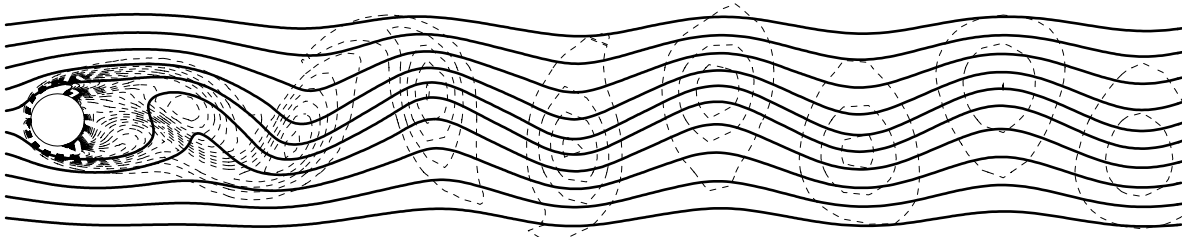

Time $=1 / 6 T$
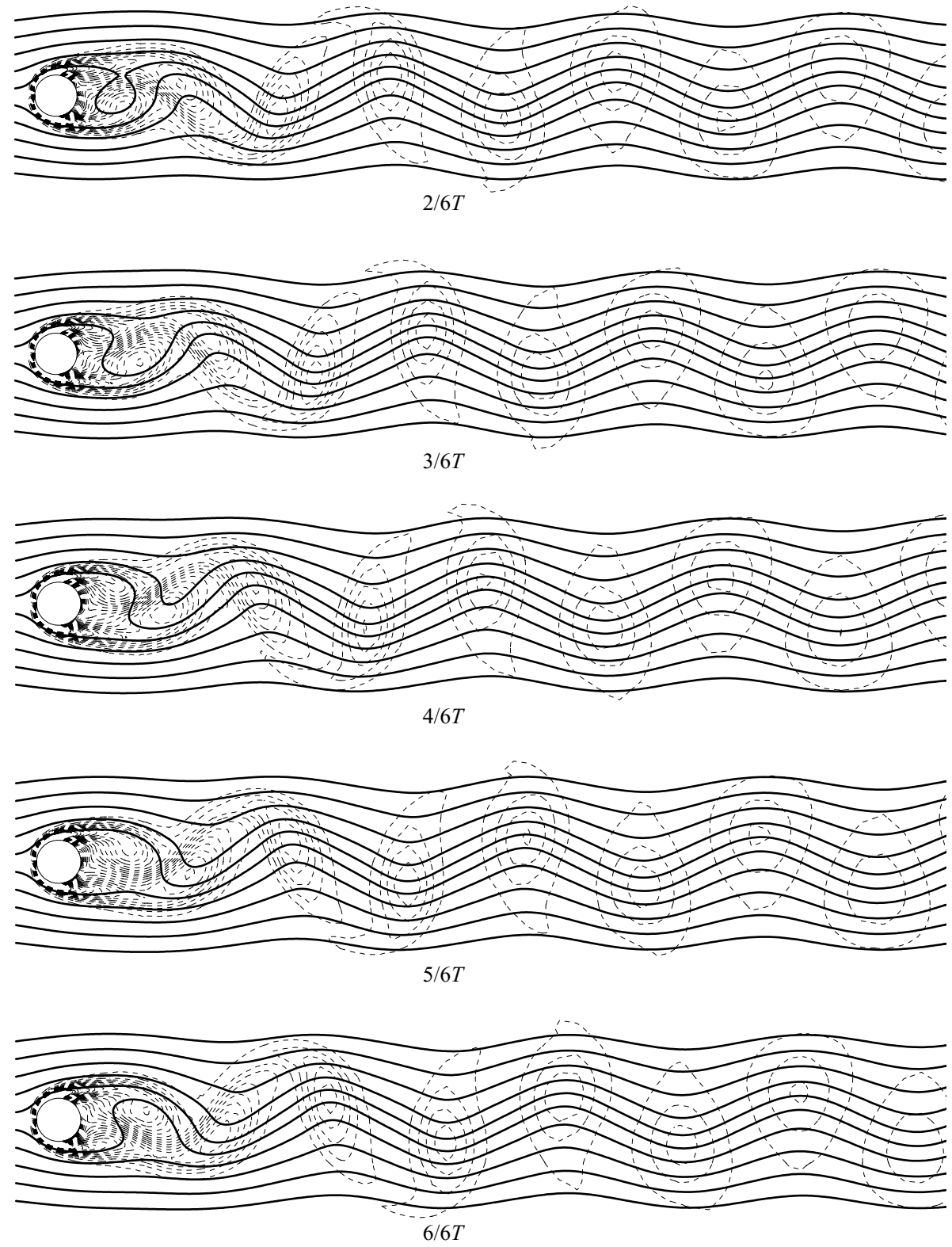

FIGURE 6. Streamlines (solid lines) and vorticity lines (dashed lines) for $R e=100$. 

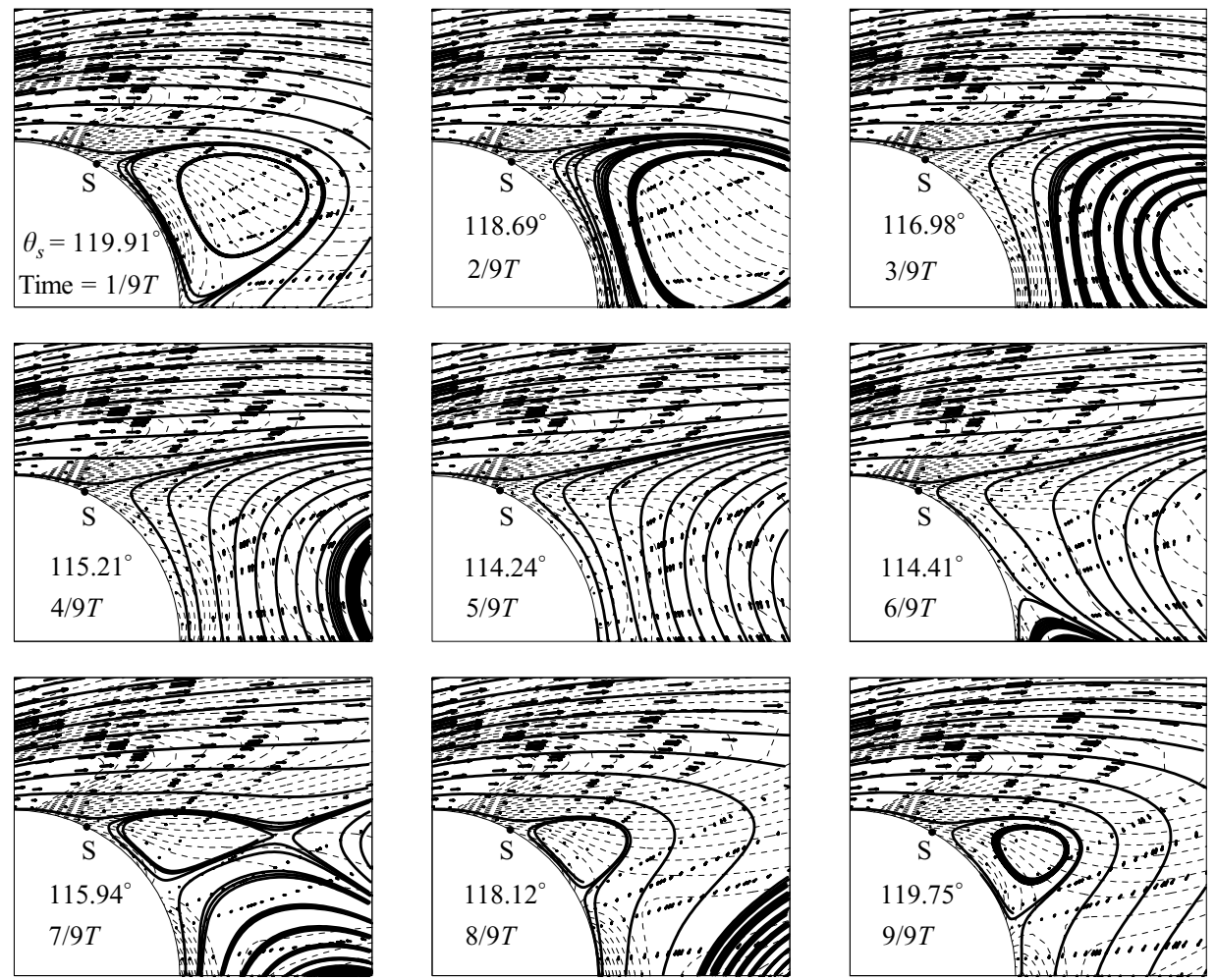

FIGURE 7. Blown-up view of streamlines, vorticity lines and velocity vectors for $R e=100$ near the separation point (denoted S).

The flow conditions are the same as in figure 6 . The velocity vectors are displayed as short arrows to indicate the flow directions in different grids, thereby showing clearly how the instantaneous separation point (denoted S, and shown as a solid point on the surface of the circular cylinder in figure 7) oscillates on the cylinder surface in a complete shedding period. The instantaneous separation angle and the corresponding instant are displayed at the lower-left corner of each plot. An oscillation of approximately $\pm 3^{\circ}$ in the separation angle is found for this flow condition.

Figure 8 shows the variations in the instantaneous separation angle $\left(\theta_{s}\right)$ of the laminar flow around a circular cylinder in a complete vortex-shedding period $\left(t_{p}\right)$ from the numerical simulations for Reynolds numbers of 50,80, 120, and 160. Since $\theta_{s}$ remains constant in the steady recirculation regime, the results for $R e<R e_{C}$ are not shown in this figure. The plot of $\theta_{s}$ versus time shown in figure 8 starts from any arbitrary instant within the vortex-shedding period. The $\theta_{s}$ value decreases with increasing Reynolds number; however, the variation in $\theta_{s}$ values increases significantly as the Reynolds number increases for $R e>R e_{C}$. As shown in the examples, the variations in the instantaneous $\theta_{s}$ values from the time-averaged mean values are around $\pm 0.5^{\circ}$ for $R e=50$, and $\pm 7^{\circ}$ for $R e=160$.

As part of the experimental results, figures $9(a)$ and $9(c)$ present two typical flash photographs of macroscopic flow patterns in the soap film for $R e=35$ and 100, respectively. The flow is left to right. Figures $9(b)$ and $9(d)$ show the corresponding flow visualization results from the towing tank. The diameter of the cylinder is $8 \mathrm{~mm}$. The shutter speed was set at 0.134 and 0.067 seconds, respectively. At lower $\operatorname{Re}$ (as shown 


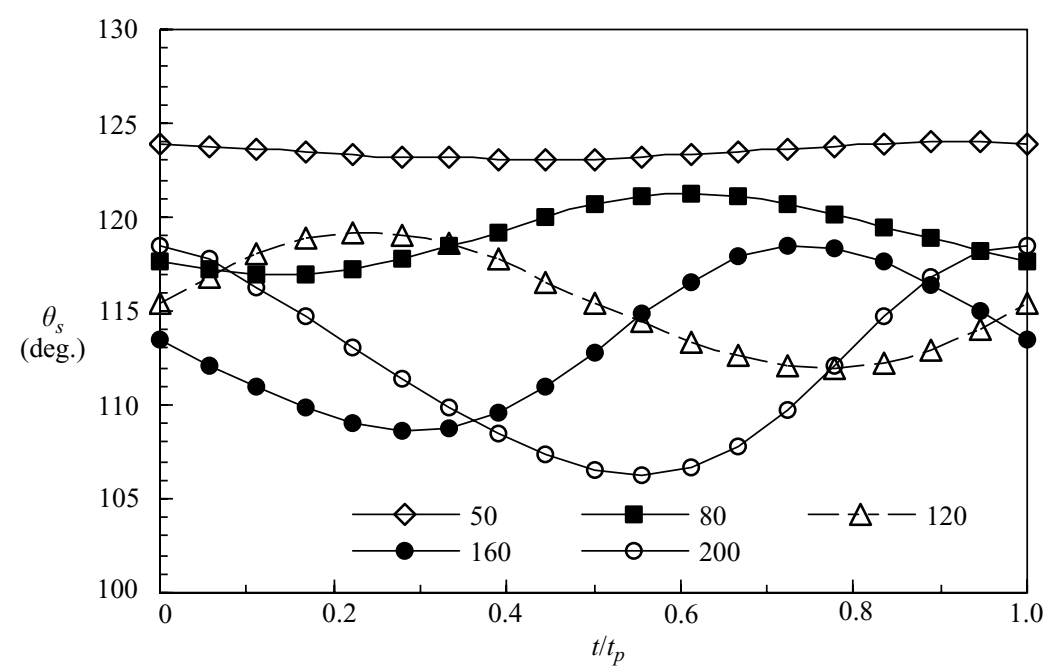

FIGURE 8. Instantaneous separation angles within a vortex-shedding period for five different Reynolds numbers from the present numerical calculations.

in figures $9 a$ and $9 b$ ), a pair of steady eddies could be seen in the wake. The separation point in the soap-film experiment was determined from the intersection of the cylinder surface and the extrapolated line from the boundary of the outermost closed fringe of the recirculation eddy (shown as the white triangle in figure $9 a$ ). As $R e$ increases, the wake becomes unstable, thus producing the von-Kármán vortex street (see figures $9 c$ and $9 d$ ). The flow features of the soap-film experiments (figures $9 a$ and $9 c$ ) are basically the same as their three-dimensional counterpart at the same $R e$ (figures $9 b$ and $9 d$ ). The separation point in the soap-film experiment was estimated near the point where the dark streakline leaves the cylinder surface, shown as the white triangle in figure $9(c)$. The error for determination of the separation angle was estimated to be less than $\pm 1.0^{\circ}$.

It has long been expected that the vortex-shedding phenomena of a bluff body have a close relationship with the characteristics of the boundary layer at the separation point. However, it is surprising to note that there is relatively little information in the literature about the time-dependent characteristics of the separation point for the flow around a circular cylinder. It is, therefore, interesting to display how the origin of the shedding vortex, i.e. the separation point, changes with time for different Reynolds numbers. Figure 10 shows time-sequenced pictures of the variation in the separation angle taken by a high-speed CCD camera from the horizontal soap-film tunnel over a shedding cycle. The camera was oriented vertically above the cylinder. The diameter of the stainless steel circular cylinder was $2.9 \mathrm{~mm}$, the shutter speed was $500 \mathrm{~Hz}$, and the frame rate was 500 frames per second. The flow was from left to right, and $R e=100$. The artificial grey portion plotted at the upper-left corner in figure $10(a-e)$ is the circular cylinder, which was measured from the reference photo taken from the same experimental setup, but without the soap film. The black rim around the cylinder was caused by the total reflection of the illuminating light incident in the meniscus region of the soap film that prevents the scattered light from reaching the camera. The width of the meniscus, denoted as $m$, was estimated to be about the same width as the black rim and was approximately $0.3-0.4 \mathrm{~mm}$ wide in the present study. Taking into account the existence of the meniscus around the cylinder, 
(a)

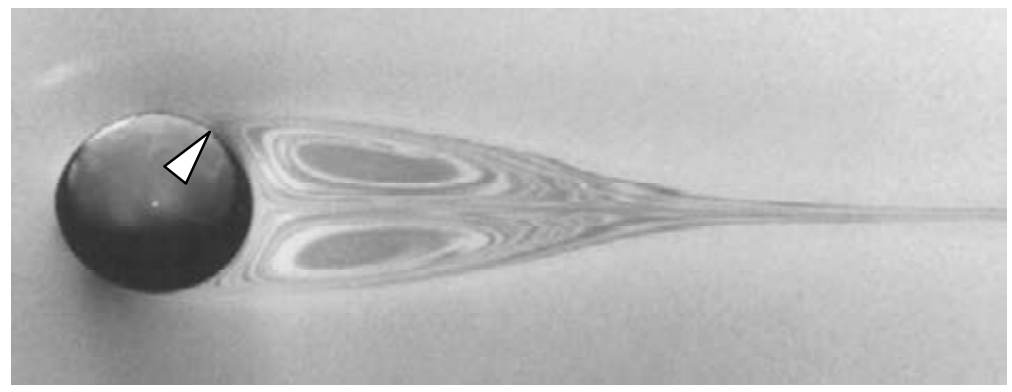

(b)

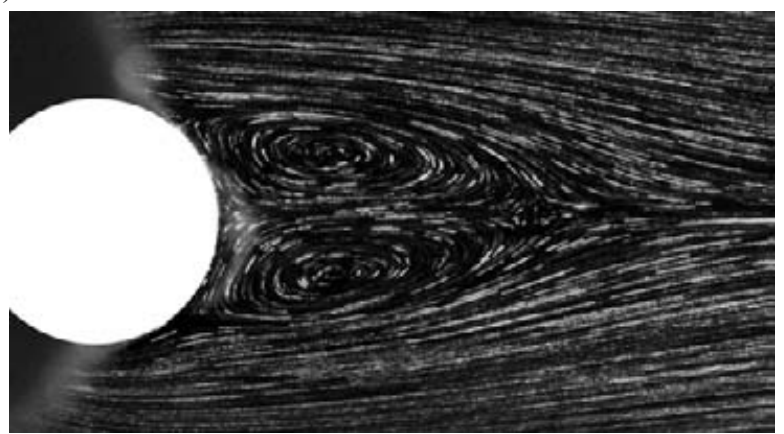

$(c)$

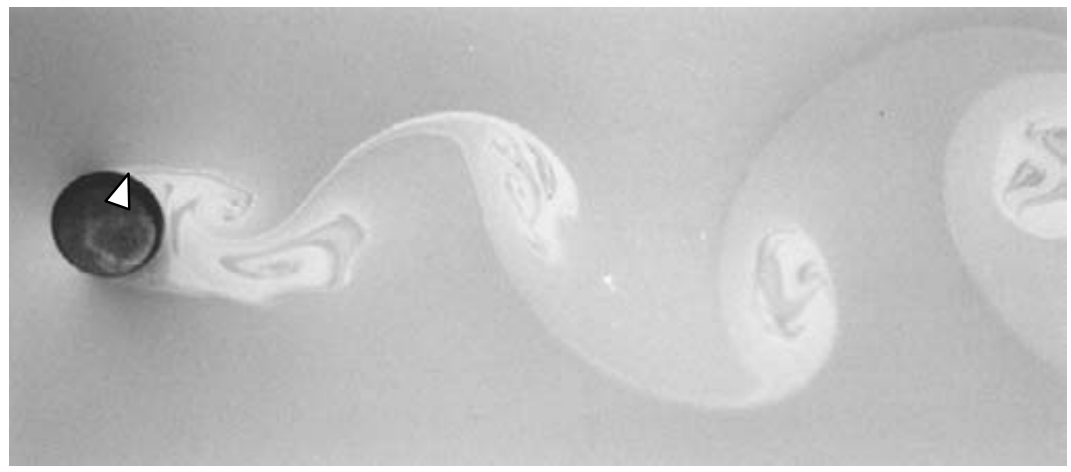

$(d)$

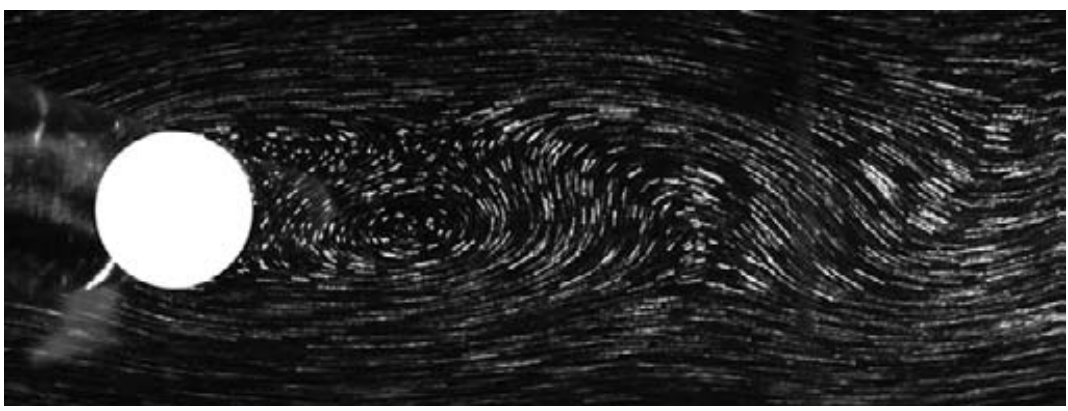

FIGURE 9. Typical flow visualization pictures of flow over a circular cylinder at $R e=35$ ( $a$ and $b)$ and $R e=100(c$ and $d) ;(a)$ and $(c)$ are from soap-film tunnels, $(b)$ and $(d)$ from the towing tank. 

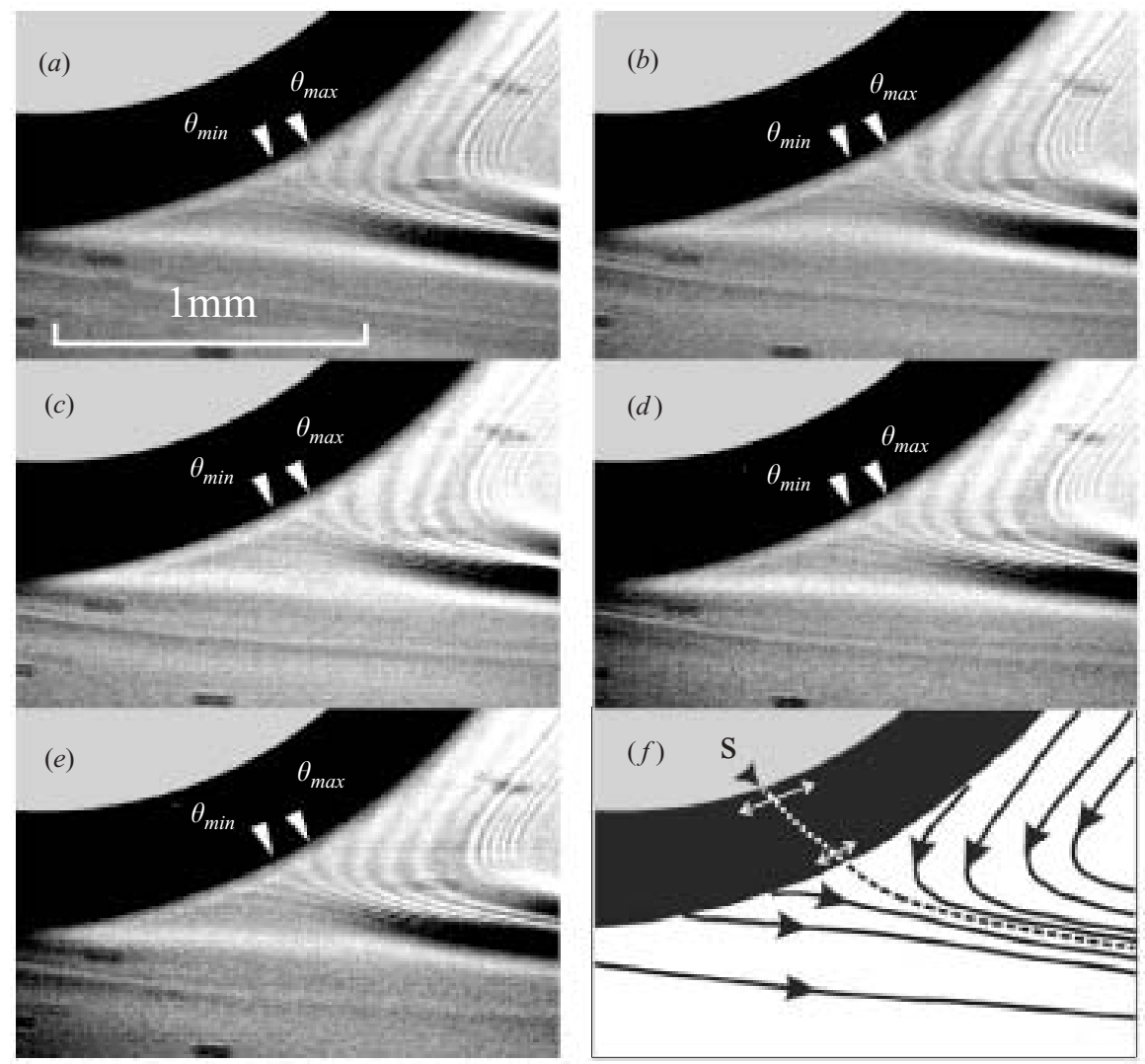

FiguRE 10. A close-up view of flow separation from a circular cylinder at $R e=100$ in the horizontal soap-film tunnel; dimensionless time in a vortex shedding cycle, $T^{*}=t / t_{p}=(a) 0$, (b) $0.227,(c) 0.5,(d)$ 0.727, and $(e)$ 1.0. In $(f)$ the flow structure is sketched (symbol $\mathrm{S}$ is separation point; white arrowheaded lines demonstrate oscillations of the separation point; solid black lines are instantaneous streamlines; dashed line is flow-dividing line).

Couder \& Basdevant (1986) defined an effective diameter, equal to $d+2 m$, as the characteristic length for calculating the Reynolds number. However, the $m$ value is not a constant, but a function of the surface contact angle between the fluid and solid surface, cylinder diameter, Reynolds number, etc. This value must be determined from each experiment. Because the flow is almost stagnant inside the meniscus from experimental observations and the meniscus has been shown to have little influence on the vortex-shedding characteristics in recent studies, e.g. Gharib \& Derango (1989), Vorobieff \& Ecke (1999), Horváth et al. (2000), and Wen \& Lin (2001), the effect of the meniscus has been absorbed into the derivation process of the film viscosity, for the sake of simplicity. This means that the cylinder diameter $D$ is still used as the characteristic length scale for the soap-film experiments, in which the film viscosity is calculated from the existing empirical Strouhal-Reynolds numbers in equation (3.1).

Figure $10(f)$ is a sketch of the representative flow pattern in figure $10(a-e)$. As described previously, the separation angle $\theta_{s}$ is determined from the position of $\mathbf{S}$, i.e. the intersection of the extrapolation line of the outermost closed fringe and the cylinder surface. Due to the existence of the dark ring caused by the meniscus, instead of $\theta_{s}$, only $\theta_{\max }$ and $\theta_{\min }$ are indicated as the white arrows on the edge of the 
black meniscus ring in figure $10(a-e)$. Here, $\theta_{\max }$ and $\theta_{\min }$ are the maximum and the minimum angles where the line of the outermost closed fringe intersects with the dark rim, as determined from the continuous experimental recording $(2 \mathrm{~s})$. The difference between $\theta_{\max }$ and $\theta_{\min }$ was estimated to be about $3^{\circ}$. Of course, from the extrapolation characteristics (see figure $10 f$ ), the oscillation of the separation angle should be larger than the oscillation range of $\left|\theta_{\max }-\theta_{\min }\right|$. As a result, figure 10 reveals clearly that, although the variation in the $\theta_{s}$ values is not large, the location of the separation dividing line, shown as the dashed line and displayed only in figure $10(f)$, has changed from one photograph to another. In any case, figure 10 shows at least a first qualitative view of the flow characteristics very near the separation point.

\subsection{Variation in separation angle with Reynolds number}

Figure 11 shows the numerically simulated flow fields near the circular cylinder for Reynolds numbers ranging from 10 to 200. The flow is from left to right. As in figure 7, only the upper-right quarter of the cylinder is plotted. The solid lines, dashed lines and short arrows are the instantaneous streamlines, vorticity lines and velocity vectors, respectively. Since the purpose of figure 11 is to reveal the variations in the separation angles with each Reynolds number, the flow field at an arbitrary instant in a shedding cycle for each Reynolds number is shown. The instantaneous $\theta_{s}$ and the corresponding $R e$ are shown within the cylinder at the lower-left corner of each figure. Figure 11 demonstrates clearly that the separation point, shown as the solid dot on the cylinder surface and denoted S, moves upstream with an increasing Reynolds number. The tendency for upstream shifting of the separation point for an increasing $R e$ is relatively small for $R e \geqslant R e_{C}$ in comparison with that for $R e<R e_{C}$.

Figure 12 shows the relationship between the time-averaged separation angle for flow around a circular cylinder and the Reynolds number. The present numerical results are shown as the solid circles with vertical error bars. Each error bar indicates the maximum oscillation range of the instantaneous separation angle around the individual time-averaged value for a given Reynolds number. Experimental data (only the time-averaged values) from different authors in the literature are also shown in this figure. All the data reveal that the time-averaged separation angle decreases as the Reynolds number increases, implying that the flow separates earlier from the cylinder wall when the Reynolds number increases for $R e \leqslant 200$. The decreasing of the separation angle with an increase in the Reynolds number is especially significant in the steady recirculation regime, i.e. $R e<\operatorname{Re}_{C}(=47.5)$ and is relatively small in the periodic wake regime, i.e. $R e \geqslant R e_{C}$. This trend agrees with that of figure 11 .

As already mentioned in the Introduction and shown again in figure 12, the distribution of the experimental data from different authors can be split into two groups. The lower data set (shown as filled grey symbols) includes experimental results from Thom (1933), Homann (1936) and Taneda (1956), and the upper data set (shown as open symbols) includes those of Grove et al. (1964) and Dimopoulos $\&$ Hanratty (1968). A band of about $10^{\circ}$ deviation in $\theta_{s}$ values exists between these two data groups. The present numerical data overlap quite well with the lower set of the experimental data in the steady recirculation regime, for $R e<R e_{C}$ (shown on the left-hand side of figure 12). The reason the data of Grove et al. (1964) lie too high in this regime is possibly due to the buoyancy effect caused from heating of the cylinder, which was used to identify the separation positions. As the Reynolds number increases, the buoyancy effect becomes less significant and the aforementioned data are in better agreement with the present results, as shown on the right-hand side of figure 12. In the flow regime of a periodic laminar wake $\left(R e \geqslant R e_{C}\right)$, the present data 
The separation angle for flow around a circular cylinder

(a)

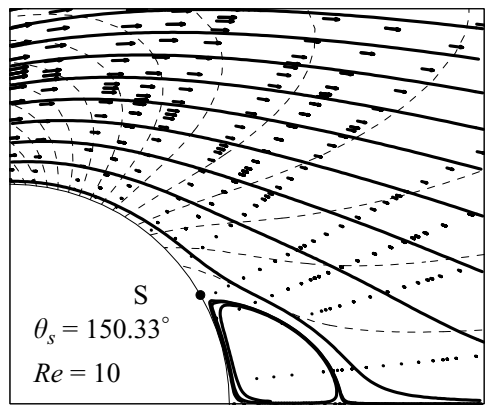

(c)

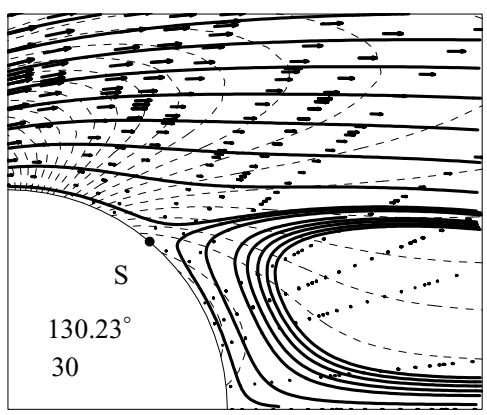

(e)

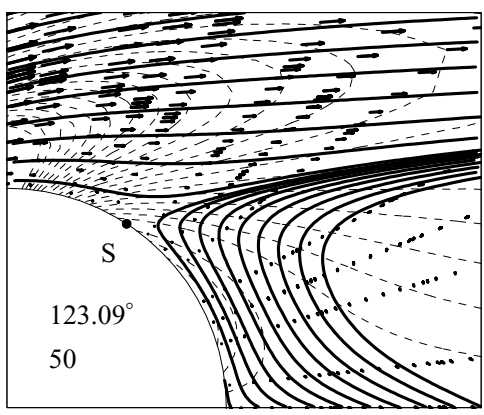

(g)

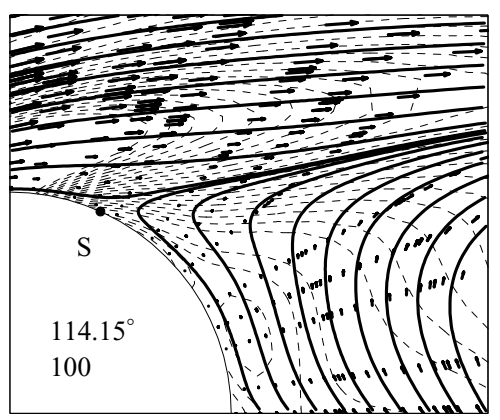

(i)

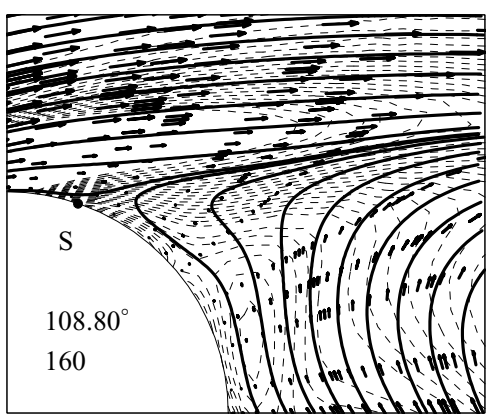

(b)

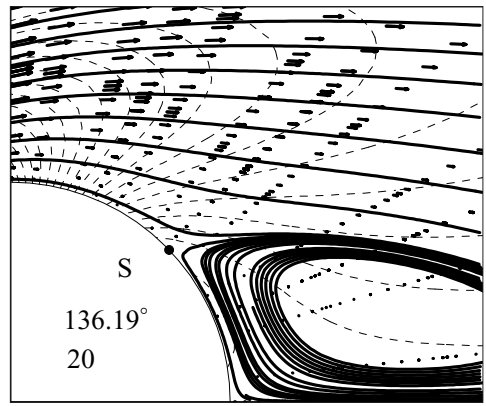

(d)

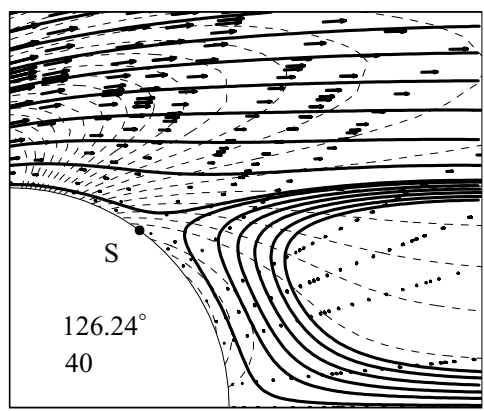

(f)

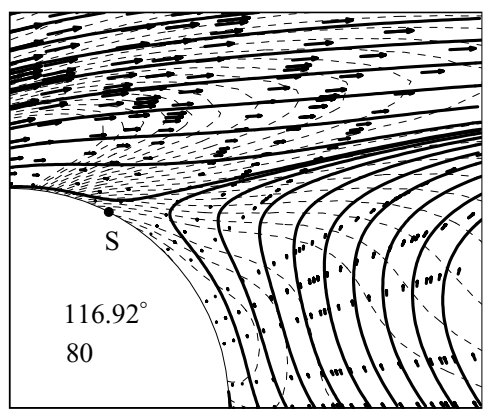

(h)

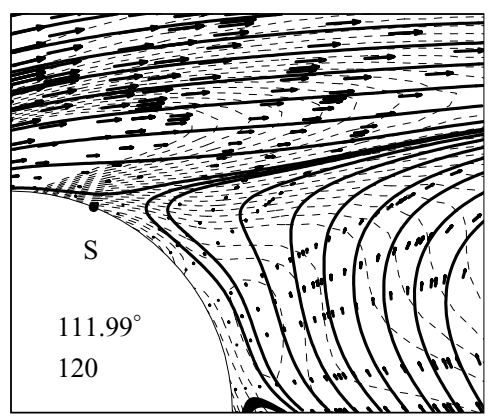

(j)

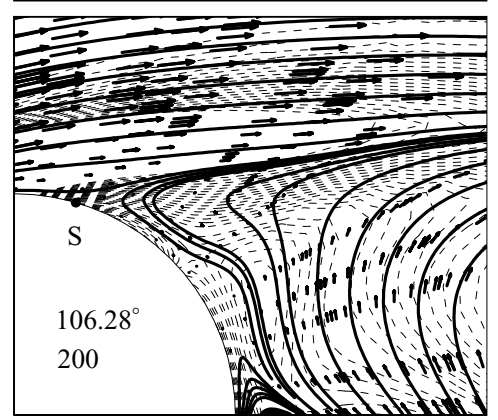

FIGURE 11. Variations in flow patterns for $R e=10$ to 200 from numerical simulations. 


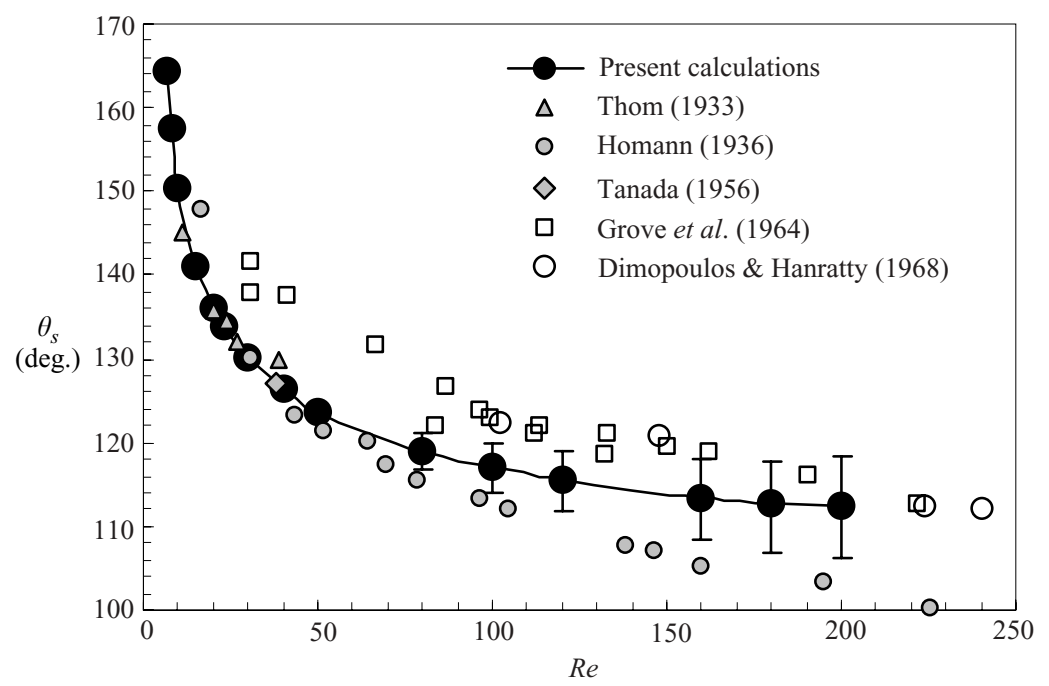

FiguRE 12. Comparisons of present numerical results with experimental data from the literature for the time-averaged separation angle and Reynolds number relationship.

begin to deviate from the lower experimental data set and approach the mean values of the upper and lower data sets as the Reynolds number increases. It is important to point out that the variation in the instantaneous separation angle with time is greater for a higher $R e$ (see the size of error bars across the solid circles), due to the oscillating character of the vortex-shedding process. The sizes of the error bars are approximately the same as the existing differences between the upper and lower experimental data sets. This means that the oscillation band precisely matches the deviation band in the literature. On the basis of the above observations from figure 12, one can reasonably conclude that the data from Thom (1933), Homann (1936) and Taneda (1956) coincide with the lower bound, and that of Grove et al. (1964) and Dimopoulos \& Hanratty (1968) coincide with the higher bound of the separation angles.

Factors such as the perspective effect of angle measurements, different limitations of the experimental methods, and the uncertainties in the unsteady flow phenomena are believed to cause this experimental deviation. In general, almost all the experimental separation angles were determined from the flow visualizations (e.g. streaklines, or pathlines of the tracers) within finite exposure times. When the tracers were released from upstream of the cylinder, large particles, which scattered much more light, revealed the large-scale motions of the flow pattern. In most cases, the large tracers seldom reached the small oscillating separation regions. Moreover, the scattering light from small tracers near the separation regions was relatively weak and was usually suppressed by the strong reflected and refracted light from the cylinder wall. Therefore, investigators might take only the nearest streakline or pathline from upstream around the cylinder to estimate the separation position. The separation angles have thus been underestimated (shifted upstream) and have typically formed the lower bound of the measured values. The case of releasing tracers from the leeside of the cylinder that forms the upper-band data is similar. On the basis of this viewpoint and considering the experimental measuring uncertainties, a quite good consistency of the present calculations with the experiments shown in figure 12 is observed. On the other hand, the data in figure 12 can explain quite well the existing deviations in the 

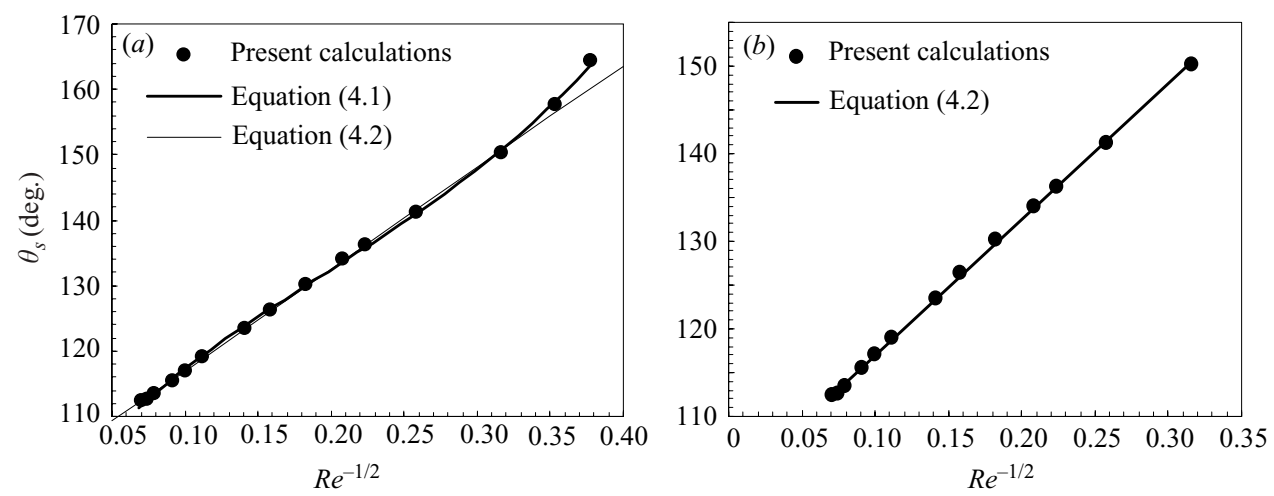

FIGURE 13. Curve fitting for the $\theta_{s}-R e^{-1 / 2}$ relationship $(a)$ by polynomial fitting for $7 \leqslant R e \leqslant 200$ and $(b)$ by linear fitting for $10 \leqslant R e \leqslant 200$.

experiments in the literature that at present remain unclear. This also suggests that the deviations in the experiments in the literature are not caused by flow bifurcation. Note that the values of the blockage ratios are 0.2 for Grove et al. (1964), about 0.1 for Homann (1936) and Thom (1933), about 0.08 for Dimopoulos \& Hanratty (1968), and less than 0.03 for Taneda (1956). However, the data from Homann (1936), Thom (1933) and Taneda (1956) belong to the same data set (lower group) in figure 12 but those from the other two studies belongs to another data set. It is very clear that no direct correlation exists between these two data sets and their corresponding blockage ratios. As a result, the experimental deviations between different researchers in the periodic wake regime must come mainly from the methodologies and criteria used, rather than from the influence of the blockage ratio as suspected previously.

\subsection{Empirical equation for separation angle and Reynolds number relationship}

The relationships between the wake characteristics and the Reynolds number have been frequently expressed in terms of $1 / R e$, e.g. the Strouhal and Reynolds number relationship by Roshko (1954) and the drag coefficient and Reynolds number relationship by Henderson (1995). However, as recently demonstrated by Fey, König \& Eckelman (1998) and Williamson \& Brown (1998), the parameter $1 / R e^{1 / 2}$ is a more appropriate than $1 / R e$ to represent the Strouhal and Reynolds number relationship for the cylinder wake. Wang et al. (2000) revealed recently that, based on an effective temperature concept, the parameter $1 / R e^{1 / 2}$ is still the most appropriate one to describe the Strouhal and effective Reynolds number relationship for flow around a heated circular cylinder. Therefore, the relationship between the separation angle and the Reynolds number is presented in the form $\theta_{s}-R e^{-1 / 2}$ in figure 13 .

Figure 13(a) shows the relationship between the averaged separation angle and the Reynolds number from various numerical calculations. The symbols and the solid line designate the calculated data and the corresponding fitting curve, respectively. From a least-square curve fitting, the four-term $\theta_{s}-R e^{-1 / 2}$ relationship is written as

$$
\theta_{s}=95.7+267.1 R e^{-1 / 2}-625.9 R e^{-1}+1046.6 R e^{-3 / 2},
$$

which yields a root-mean-square error of 0.0004 for $7 \leqslant R e \leqslant 200$. Note that no steady separation bubble was found for $R e \leqslant 6$. This result is the same as that of Takami \& Keller (1969). 


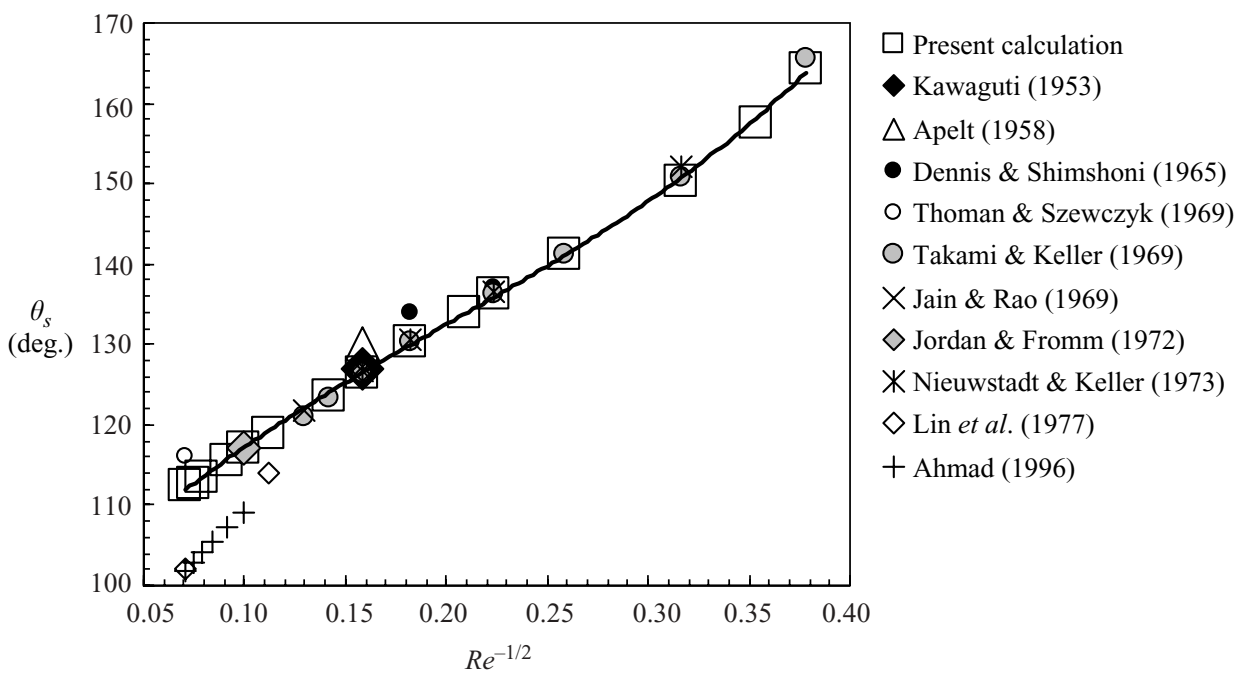

FIGURE 14. Present $\theta_{s}-R e^{-1 / 2}$ relationship in comparison with numerical data from the literature; equation (4.1) is plotted as a solid line.

If the fitting range is restricted to $10 \leqslant R e \leqslant 200$, a linear empirical equation for the $\theta_{s}-R e^{-1 / 2}$ relationship is obtained as

$$
\theta_{s}=101.5+155.2 R e^{-1 / 2}
$$

which yields a root-mean-square error of 0.0005 . This means that the $\theta_{s}-R e^{-1 / 2}$ relationship curve, shown in figure 13(b), is quite linear except for the very low Reynolds number range, i.e. $\operatorname{Re}<10$. Also shown in figure $13(a)$ is the dashed curve of (4.2) for comparison with (4.1). Since the dimensionless boundary layer thickness and the Strouhal and Reynolds number relationship for the circular cylinder are also proportional to $R e^{-1 / 2}$ (Fey et al. 1998; Williamson \& Brown 1998; Wang et al. 2000), (4.2) provides direct evidence for the linkage of the two phenomena. The variation in vortex shedding is governed by the flow characteristics at the separation position, the latter being characterized by the boundary layer flow on the cylinder surface.

Figure 14 shows comparisons of the numerical results from different authors (shown as symbols) and the empirical equation (4.1) (shown as a solid curve). The present numerical data are also plotted as open square symbols in this figure, which shows that most numerical data from different investigations fall on the (4.1) curve, except the data from Lin et al. (1977) and Ahmad (1996). The latter used the steady-state approach of the Navier-Stokes equation to solve the problem in the region $R e>R e_{C}$. Overall, a quite good consistency of the present calculations with most of the numerical work is revealed. On the other hand, the applicability of (4.1) in the entire laminar range is also revealed.

Figure 15(a) represents the variation in the measured time-averaged separation angle with the Reynolds number from the soap-film experiments. The mean value of the experimental separation angle was taken from ten randomly frozen instantaneous photographs (by using $1.2 \mu$ s illumination) at a given Reynolds number. Again, the error bars show the maximum oscillation ranges of the instantaneous measured values from the averaged separation angles. Also shown in figure 15(a) for comparison are the data from the particle-streak visualization in the towing tank. Excellent agreement between the measured data and the numerical results is observed in this figure. 

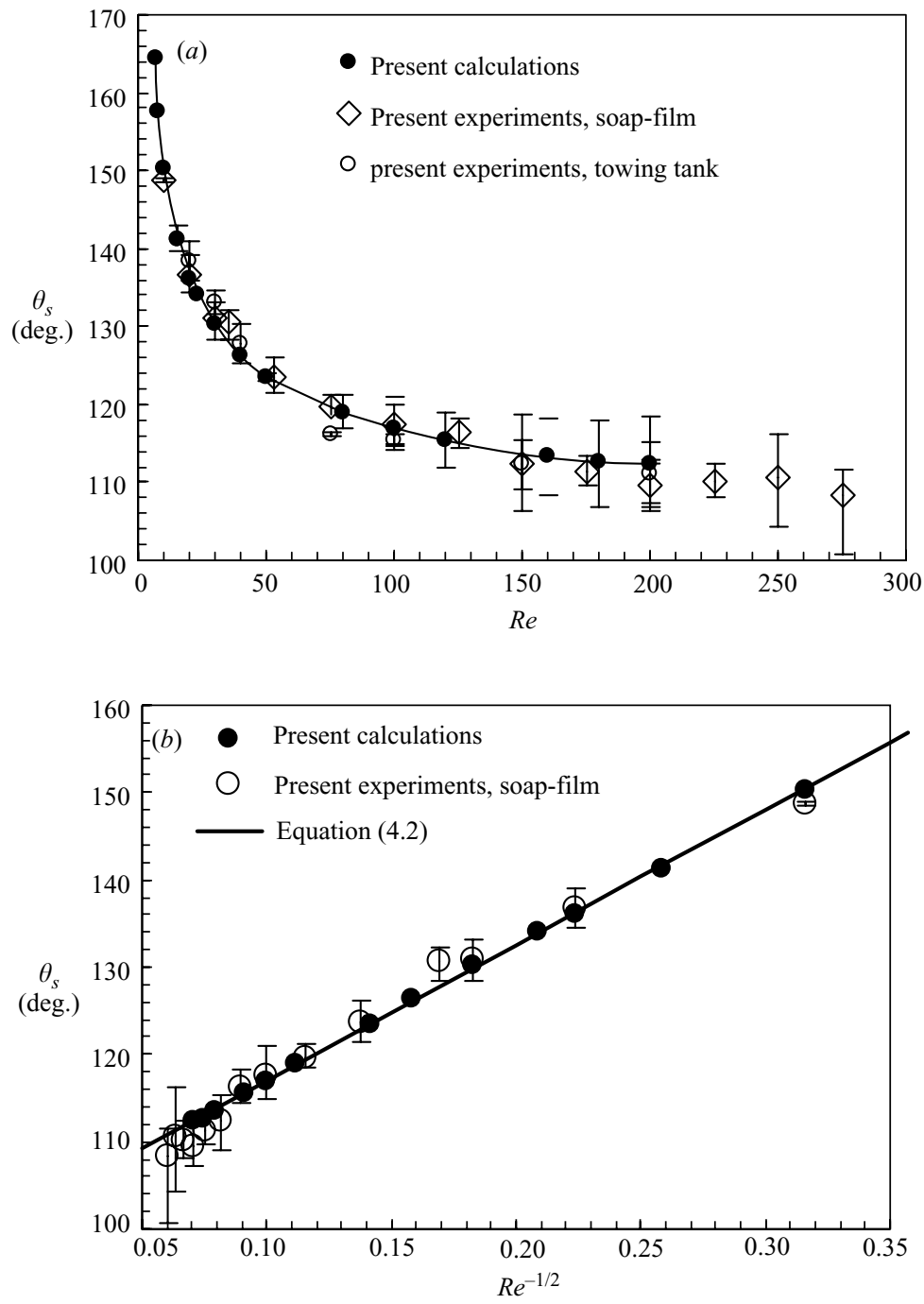

FIGURE 15. Comparison of separation angles of present experiments and numerical simulations: (a) $\theta_{s}-R e$ plot and $(b) \theta_{s}-R e^{-1 / 2}$ plot.

Figure $15(b)$ shows an alternative presentation of figure $15(a)$ in the form of the $\theta_{s}-R e^{-1 / 2}$ relationship. Equation (4.2) is also plotted in the figure. Again, excellent agreement among the measured data from the soap-film experiments, the numerical results and (4.2) is demonstrated.

As mentioned in $\S 3.1$, the blockage ratio of a cylinder, denoted $B R$, is defined as the ratio of the cylinder diameter to the channel width between the top and bottom walls (with a no-slip condition). For the numerical simulations, if the free-stream boundary condition is applied at both the top and bottom walls as shown in figure $2(a)$, this is denoted the case of zero blockage ratio and is considered as the comparison standard that free from the blockage effect. Figure 16 shows a comparison of numerical and experimental results of time-averaged separation angles for different blockage ratios at $R e=10$ and 30. The reason to choose these Reynolds numbers is because values of the separation angle are higher in the steady recirculation regime and the change due 


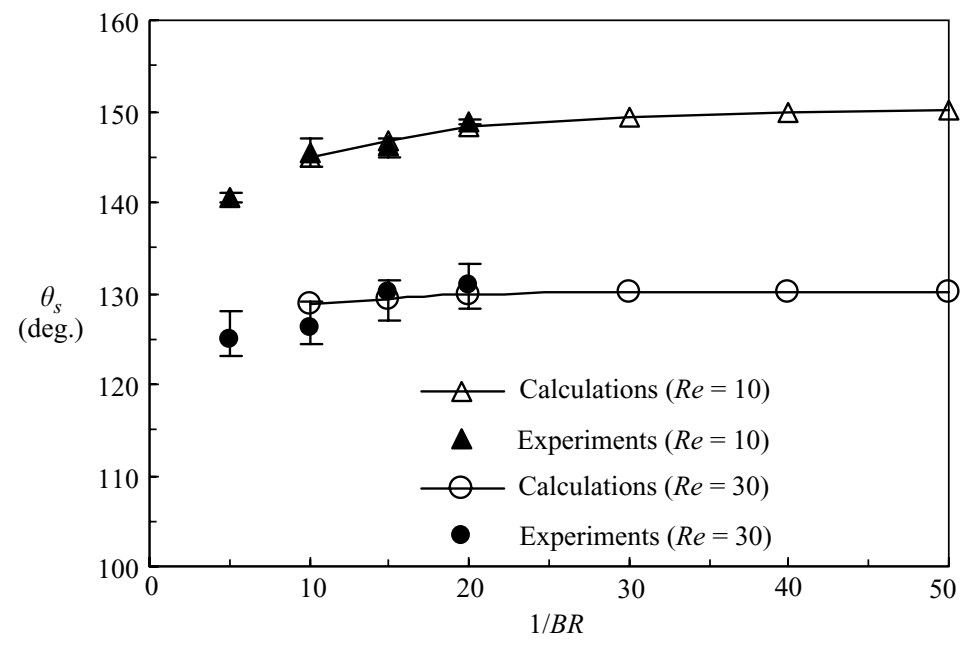

FIGURE 16. Comparison of time-averaged separation angle from experiments and numerical simulations for different blockage ratios $(B R)$.

to blockage ratio could be thus easily detected by experiments. The mean value of the experimental separation angle was taken from nine independent runs at the given Reynolds numbers. The error bars show the maximum variation of the measured values of the separation angles. Very good agreement between the numerical data and the experimental results is observed in this figure. Moreover, the higher the blockage ratio is, the lower the value of the separation angle becomes. This could be interpreted physically as the acceleration effect of the flow around a circular cylinder becoming more significant when the two sidewalls approach to the cylinder. The local Reynolds number thus becomes larger and the flow separates earlier from the cylinder in comparison with that of lower $B R$-values.

To obtain more quantitative information on the effect of blockage ratio on the separation angle, figure 17 shows the simulation results of the time-averaged separation angle at different blockage ratios in both of the steady recirculation and laminar vortex-shedding regimes. Figure 17( $a)$ shows the difference of time-averaged separation angle $\Delta \theta_{s}$ (in degrees) between $\theta_{s}(R e, B R)$ and $\theta_{s}(R e, B R=0)$. Figure $17(b)$ displays the dimensionless form of the separation angle $\theta_{s}^{*}$, which is defined as the ratio of $\theta_{s}(R e, B R)$ and $\theta_{s}(R e, B R=0)$. For comparison, the case without blockage effect is shown as the dashed lines in figure 17. The figure reveals clearly that the deviation of the time-averaged separation angle from $\theta_{s}(R e, B R=0)$ becomes more significant as the blockage ratio increases in all calculated cases. In figure 17(a) the higher the Reynolds number is, the smaller the value of $\Delta \theta_{s}$ is for the same blockage ratio. For the cases in the periodic wake regime, e.g. $R e=120$ and 160 , all values of $\Delta \theta_{s}$ are less than $0.5^{\circ}$ for $B R=1 / 10$, which is commonly considered as the maximum allowable blockage ratio for the two-dimensional experiments. This shows clearly that the value of $\Delta \theta_{s}$ caused by the blockage effect is one order of magnitude less than the well-known deviation $\left(\sim 10^{\circ}\right.$ see figure 1$)$ of the upper and lower data sets from various articles in the literature. Regarding the blockage effect on the oscillation of the separation angle (not shown), this is less than $0.8^{\circ}$ for $B R=1 / 10$ at $R e=120$ and 160. The influence of the blockage effect is also not significant. Therefore, present results reveal directly and clearly that the blockage effect is not the cause of the 

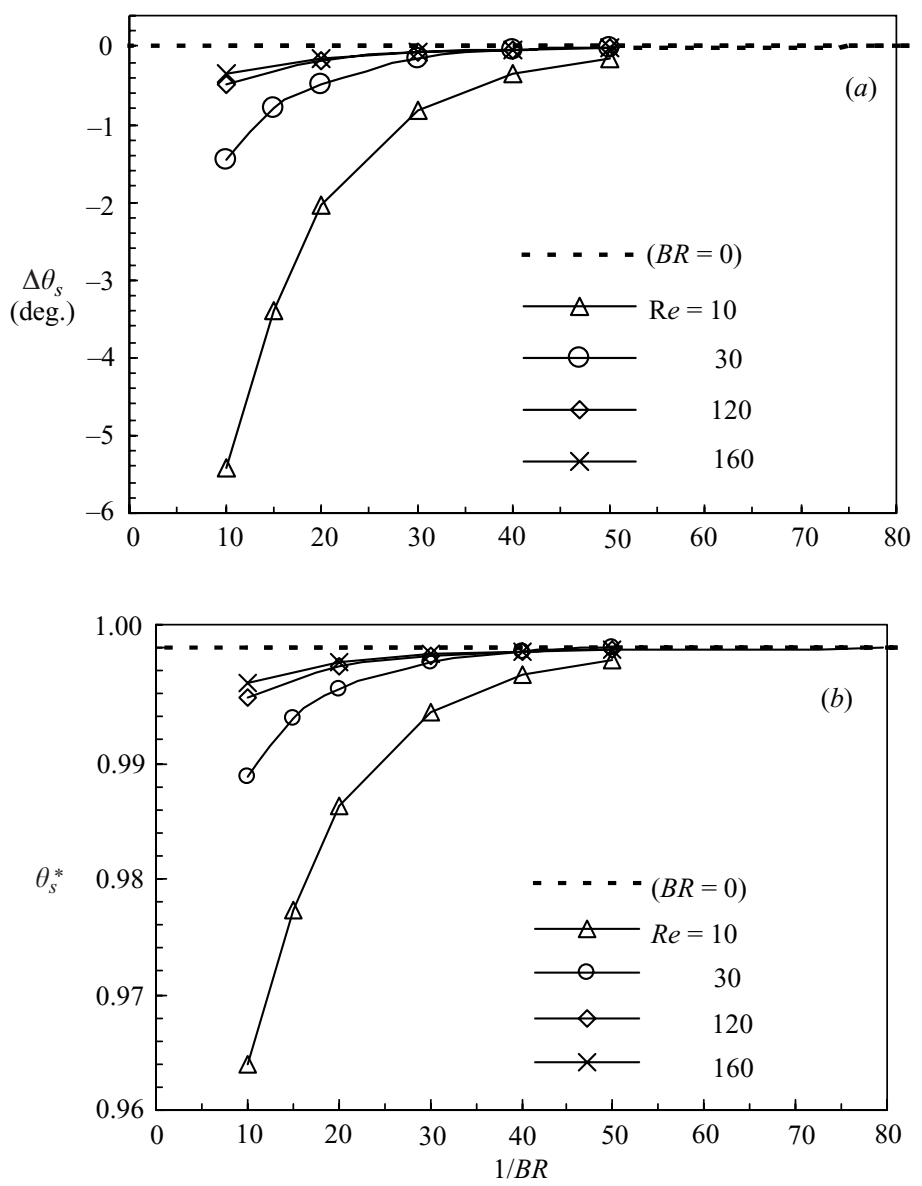

FIGURE 17. Relationship between time-averaged separation angle and blockage ratio $(B R)$ from simulations: $(a) \Delta \theta_{s}-B R$ plot and $(b) \theta_{s}^{*}-B R$ plot, where $\theta_{s}^{*}$ is the separation angle normalized by that without a blockage effect (the unbounded flow, i.e. the top and bottom are at in the free-stream boundary condition).

discrepancy in figure 1 . The blockage effect has more significant effect on the timeaveraged separation angle in the steady recirculation regime than that in the laminar vortex-shedding regime. For instance, $\Delta \theta_{s}$ reaches about $5.5^{\circ}$ and $1.5^{\circ}$ for $B R=1 / 10$ at $R e=10$ and 30, respectively. As a result, the blockage effect has much more significant influence on the separation angle for lower Reynolds number. However, this is still within the measuring uncertainty for most experiments and, moreover, the numerical and experimental data of previous studies are basically consistent in this regime (see figures 12 and 14).

\section{Conclusions}

The separation angle of the flow around a circular cylinder has been numerically and experimentally studied for $R e<280$. The results from the numerical simulations have clearly revealed that the long-existing deviation band of separation angles obtained by different researchers in the literature results from the experimental methodologies and the intrinsic unsteady nature of the separation point in this Reynolds number 
range. The blockage ratio, which is the commonly suggested reason for this discrepancy among experiments, has been demonstrated to be not the major issue. For the time-averaged separation angles, the present numerical results can be well described by a four-term polynomial curve fitting of $\theta_{s}=95.88+264.76 R e^{-1 / 2}-619.01 R e^{-1}+$ $1042.4 R e^{-3 / 2}$ for the $\theta_{s}-R e^{-1 / 2}$ relationship for Reynolds numbers ranging between 7 and 200. This fitting curve gives a root-mean-square error of 0.0004 in the investigated Reynolds number range and agrees well with other numerical studies in the literature. Excluding data for $R e<10$, a linear empirical relationship has been proposed for Reynolds numbers ranging from 10 to 200 , written as $\theta_{s}=101.45+155.05 R e^{-1 / 2}$. This linear fitting curve gives a root-mean-square error of 0.0005 .

Experimentally, the soap-film flow visualization method has been used to derive the separation angle for the first time. This method offers minimum uncertainties in determining the separation angle in comparison with all existing counterparts of three-dimensional flow-visualization methods. The experimental data are in excellent agreement with the present numerical simulations.

The authors would like to thank the National Science Council of the Republic of China for financially supporting this research under Contract No. NSC 90-2212-E002-232, NSC 89-2218-E-002-107, NSC 88-2212-E-002-035 and NSC 87-2212-E-212008. Professor M. Gharib (California Institute of Technology) and Professor W. I. Goldburg (University of Pittsburgh) are appreciated for their valuable suggestions in designing the soap-film tunnels. The authors also wish to express appreciation to Dr C. Rutledge for her editorial assistance.

\section{REFERENCES}

Ahmad, R. A. 1996 Steady-state numerical solution of the Navier-Stokes and energy equations around a horizontal cylinder at moderate Reynolds numbers from 100 to 500. Heat Transfer Engng 17, 31-81.

Allen, D. N. \& Southwell, R. V. 1955 Relaxation methods applied to determine the motion in two dimensions of a viscous fluid past a fixed cylinder. Q. J. Mech. Appl. Maths 8, 129-145.

Apelt, C. J. 1958 The steady flow of a viscous fluid past a circular cylinder at Reynolds numbers 40 and 44. Aero. Res. Counc. Lond. $R \&$ M 3175, 1-28.

Barkley, D. \& Henderson, R. D. 1996 Three-dimensional Floquet stability analysis of the wake of a circular cylinder. J. Fluid Mech. 322, 215-241.

Beizaie, M. \& Gharib, M. 1997 Fundamentals of a liquid (soap) film tunnel. Exps. Fluids 23, $130-140$.

Blackburn, H. M. \& Henderson, R. D. 1999 A study of two-dimensional flow past an oscillating cylinder. J. Fluid Mech. 385, 255-286.

Chomaz, J. M. 2001 The dynamics of a viscous soap film with soluble surfactant. J. Fluid Mech. 442, 387-409.

COUder, Y. 1981 The observation of a shear flow instability in a rotating system with a soap membrane. J. Phys. Lett. 42, 429-431.

Couder, Y. 1984 Two-dimensional grid turbulence in a thin liquid film. J. Phys. Lett. 45, 353-360.

Couder, Y. \& Basdevant, C. 1986 Experimental and numerical study of vortex couples in twodimensional flows. J. Fluid Mech. 173, 225-251.

Couder, Y., Chomaz, J. M. \& Rabaud, M. 1989 On the hydrodynamics of soap films. Physica D 37, 384-405.

Coutanceau, M. \& Bouard, R. 1977 Experimental determination of the main features of the viscous flow in the wake of a circular cylinder in uniform translation. Part 1. Steady Flow. J. Fluid Mech. 79, 231-256.

Coutanceau, M. \& Defaye, J. 1991 Circular cylinder wake configurations: A flow visualization survey. Appl. Mech. Rev. 44(6), 255-305. 
Dennis, S. C. R. \& Shimshoni, M. 1965 The steady flow of a viscous fluid past a circular cylinder. Aero. Res. Counc. Current Papers, no. 797.

Dimopoulos, H. G. \& Hanratty, T. J. 1968 Velocity gradients at the wall for flow around a cylinder for Reynolds numbers between 60 and 360. J. Fluid Mech. 33, 303-319.

Eisenlohr, H. \& EcKelmann, H. 1989 Vortex splitting and its consequences in the vortex street wake of cylinders at low Reynolds numbers. Phys. Fluids A 1, 189-192.

Fey, U., KöNIG, M. \& ECKelmann, H. 1998 A new Strouhal-Reynolds-number relationship for the circular cylinder in the range $47<R e<2 \times 10^{5}$. Phys. Fluids 10, 1547-1549.

Gharib, M. \& Derango, P. 1989 A liquid film (soap film) tunnel to study two-dimensional laminar and turbulent shear flows. Physica D 37, 406-416.

Grove, A. S., Shair, F. H., Petersen, E. E. \& Acrivos, A. 1964 An experimental investigation of the steady separated flow past a circular cylinder. J. Fluid Mech. 19, 60-81.

Hammache, M. \& Gharib, M. 1991 An experimental study of the parallel and oblique vortex shedding from circular cylinders. J. Fluid Mech. 232, 567-590.

Henderson, R. D. 1995 Details of the drag curve near the onset of vortex shedding. Phys. Fluids 7, 2102-2104.

Henderson, R. D. 1997 Nonlinear dynamics and pattern formation in turbulent wake transition. J. Fluid Mech. 352, 65-112.

Homann, F. 1936 Einfluss grsser Zähigkeit bei Strmung um Zylinder. Forsch. Ing. Wes. 7, 1-9.

Horváth, V. K., Cressman, J. R., Goldburg, W. I. \& Wu, X. L. 2000 Hysteresis at low Reynolds number: Onset of two-dimensional vortex shedding. Phys. Rev. E 61(5), R4702-R4705.

JaIn, P. C. \& SANkara RaO, K. 1969 Numerical solution of unsteady viscous incompressible fluid flow past a circular cylinder. Phys. Fluids Suppl. II, 57-64.

Jordan, S. K. \& Fromm, J. E. 1972 Oscillating drag, lift and torque on a circular cylinder in a uniform flow. Phys. Fluids 15, 371-376.

Karniadakis, G. E. \& Triantafyllou, G. S. 1992 Three-dimensional dynamics and transition to turbulence in the wake of bluff bodies. J. Fluid Mech. 238, 1-30.

Kawaguti, M. 1953 Numerical solution of the Navier-Stokes equations for the flow around a circular cylinder at Reynolds number 40. J. Phys. Soc. Japan 8, 747-757.

Kawaguti, M. \& JaIn, P. 1965 Numerical study of a viscous fluid flow past a circular cylinder. J. Phys. Soc. Japan 21, 2055-2061.

Kellay, H., Wu, X. L. \& GoldburG, W. 1995 Experiments with turbulent soap films. Phys. Rev. Lett. 74, 3975-3978.

Keller, H. B. 1958 On some iterative methods for solving elliptic difference equations. Q. Appl. Maths 16, 209-226.

Keller, H. B. \& Takami, H. 1966 Numerical studies of steady viscous flow about cylinders. In Numerical Solutions of Nonlinear Differential Equations (ed. D. Greenspan), p. 8. New York.

Lin, C. C., Pepper, D. W. \& LeE, S. C. 1977 Numerical methods for separated flow solutions around a circular cylinder. AIAA J. 14, 900-907.

Miller, G. D. \& Williamson, C. H. K. 1994 Control of three-dimensional phase dynamics in a cylinder wake. Exps. Fluids 18, 26-35.

MerzKirch, W. 1974 Flow Visualization. Academic.

Nieuwstadt, F. \& Keller, H. B. 1973 Viscous flow past circular cylinders. J. Fluid Mech. 1, 59-71.

Rivera, M., VorobiefF, P. \& EcKe, R. E. 1998 Turbulence in flowing soap films: velocity, vorticity and thickness fields. Phys. Rev. Lett. 81, 1417-1420.

Roshko, A. 1954 On the development of turbulent wakes from vortex streets. NACA Rep. 1191.

Rutgers, M. A., Wu, X. L., Bhagavatula, R., Petersen, A. A. \& Goldburg, W. I. 1996 Twodimensional velocity profiles and laminar boundary layers in flowing soap films. Phys. Fluids 8, 2847-2854.

TaKami, H. \& Keller, H. B. 1969 Steady two-dimensional viscous flow of an incompressible fluid past a circular cylinder. Phys. Fluids 12 Suppl II, 51-56.

TANEDA, S. 1956 Experimental investigation of the wakes behind cylinders and plates at low Reynolds numbers. J. Phys. Soc. Japan 11, 302-307.

Tном, A. 1928 An investigation of fluid flow in tow-dimensions. Aero. Res. Counc. Tech. Rep. 1194.

Тном, A. 1933 The flow past circular cylinders at low speeds. Proc. R. Soc. Lond. A 141, 651-669. 
Thoman, D. C. \& SzewczyK, A. A. 1969 Time-dependent viscous flow over a circular cylinder. Phys. Fluids 12 Suppl II, 76-86.

Trapeznikov, A. A. 1957 Application of the method of two-dimensional viscosity and shear strength to the investigation of the structure and composition of two-sided films and surface layers in solutions of soaps and saponins. Proc. 2nd Intl Congress on Surface Activity, pp. 242-258. Butterworths.

Vorobieff, P. \& Ecke, R. 1999 Cylinder wakes in flowing soap films. Phys. Rev. E 60, 2953-2956.

WANG, A. B., TrávníEK, Z. \& CHIA, K. C. 2000 On the relationship of effective Reynolds number and Strouhal number for the laminar vortex shedding of a heated circular cylinder. Phys. Fluids 12, 1401-1410.

Wen, C. Y. \& Lin, C. Y. 2001 Two-dimensional vortex shedding of a circular cylinder. Phys. Fluids 13, 557-560.

Williamson, C. H. K. 1989 Oblique and parallel modes of vortex shedding in the wake of a circular cylinder at low Reynolds numbers. J. Fluid Mech. 206, 579-627.

Williamson, C. H. K. 1996 Vortex dynamics in the cylinder wake. Annu. Rev. Fluid Mech. 28, 477-539.

Williamson, C. H. K. \& Brown, G. L. 1998 A series in $(1 / R e)$ to represent the Strouhal-Reynolds number relationship of the cylinder wake. J. Fluids Struct. 12, 1073-1085.

Wu, X., Martin, B. K., Kellay, H. \& Goldburg, W. 1995 Hydrodynamic convection in a twodimensional Couette cell. Phys. Rev. Lett. 75, 236-239.

YANG, T. S., Wen, C. Y. \& LIN, C. Y. 2001 Interpretation of color fringes in flowing soap films. Expl Thermal Fluid Sci. 25, 141-149.

Yen, R. H. \& Hsu, Y. M. 2004 Application of spectral element method with fast steady flow solver. In preparation. 